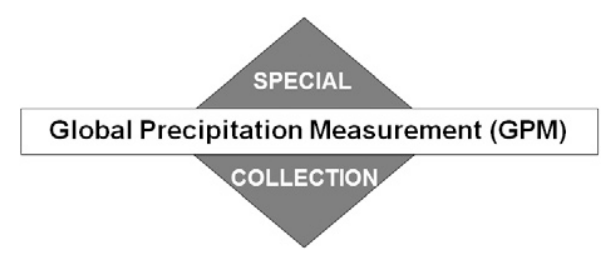

\title{
๖The Impact of Assimilation of GPM Microwave Imager Clear-Sky Radiance on Numerical Simulations of Hurricanes Joaquin (2015) and Matthew (2016) with the HWRF Model
}

\author{
ZhaOXIA PU AND ChaUlam Yu \\ Department of Atmospheric Sciences, University of Utah, Salt Lake City, Utah \\ VIJAY TALLAPRAGADA \\ Environmental Modeling Center, NOAA/NCEP, College Park, Maryland
}

JIANJUN JIN

Goddard Earth Sciences Technology and Research, Universities Space Research Center, Columbia, Maryland

WILL MCCARTY

Global Modeling and Assimilation Office, NASA Goddard Space Flight Center, Greenbelt, Maryland

(Manuscript received 14 July 2017, in final form 9 October 2018)

\begin{abstract}
The impact of assimilating Global Precipitation Measurement (GPM) Microwave Imager (GMI) clear-sky radiance on the track and intensity forecasts of two Atlantic hurricanes during the 2015 and 2016 hurricane seasons is assessed using the Hurricane Weather Research and Forecasting (HWRF) Model. The GMI clearsky brightness temperature is assimilated using a Gridpoint Statistical Interpolation (GSI)-based hybrid ensemble-variational data assimilation system, which utilizes the Community Radiative Transfer Model (CRTM) as a forward operator for satellite sensors. A two-step bias correction approach, which combines a linear regression procedure and variational bias correction, is used to remove most of the systematic biases prior to data assimilation. Forecast results show that assimilating GMI clear-sky radiance has positive impacts on both track and intensity forecasts, with the extent depending on the phase of hurricane evolution. Forecast verifications against dropsonde soundings and reanalysis data show that assimilating GMI clear-sky radiance, when it does not overlap with overpasses of other microwave sounders, can improve forecasts of both thermodynamic (e.g., temperature and specific humidity) and dynamic variables (geopotential height and wind field), which in turn lead to better track forecasts and a more realistic hurricane inner-core structure. Even when other microwave sounders are present (e.g., AMSU-A, ATMS, MHS, etc.), the assimilation of GMI still reduces temperature forecast errors in the near-hurricane environment, which has a significant impact on the intensity forecast.
\end{abstract}

\section{Introduction}

Since the 1990s, satellite observations have become a major source of observations for numerical weather prediction (NWP), owing to the rapid development of

D Denotes content that is immediately available upon publication as open access.

Corresponding author: Dr. Zhaoxia Pu, zhaoxia.pu@utah.edu radiative transfer models, data assimilation technologies, and launches of numerous major satellites. Specifically, data assimilation allows the incorporation of observational information into the NWP system if the transformation of analysis variables into the form of observations is achievable. For satellite radiance observations, this can be accomplished by using fast radiative transfer models, which simulate the satellite-observed radiances for a given atmospheric state, satellite scan geometry, and surface properties. The atmospheric state is then adjusted 
according to differences between simulated and observed radiances such that the final analysis is at the maximum likelihood (e.g., Derber and Wu 1998). Many studies have demonstrated the added benefits to NWP of directly assimilating satellite radiance observations in both global models (Derber and Wu 1998; McNally et al. 2000; Okamoto and Derber 2006; Bauer et al. 2006; Miyoshi and Sato 2007; Sakamoto and Christy 2009; Goerss 2009; Aravéquia et al. 2011; Hoppel et al. 2013) and regional models (e.g., Zou et al. 2011; Liu et al. 2012; Qin et al. 2013; M. Zhang et al. 2013; S. Q. Zhang et al. 2013; Kazumori 2014; Wang et al. 2015; Lin et al. 2017).

So far, the assimilation of radiance observations is known to be able to significantly reduce error in NWP, specifically over regions where conventional observations are sparse. For instance, hurricane forecasts, in particular, benefit greatly from the large spatial coverage over oceans and the high temporal resolution of satellite observations. This is because hurricanes form and evolve mostly over the oceans, where conventional observations such as radiosonde and surface observations are less available. Since the last decade, the research community has devoted great effort to assimilating satellite observations to improve hurricane track and intensity predictions ( $\mathrm{Pu}$ et al. 2002, 2008; Pu and Zhang 2010; Liu et al. 2012; Xu et al. 2013; Zou et al. 2013; Zhang and Pu 2014; Yang et al. 2016; Xu et al. 2016; Wu et al. 2016). Specifically, these previous studies found that satellite microwave sounders is particularly useful for understanding moist processes associated with hurricanes owing to its unique capability to depict precipitation structure and moisture processes.

The Global Precipitation Measurement (GPM) is a recent constellation-based satellite mission with microwave imagery that was initiated by the National Aeronautics and Space Administration (NASA) and the Japan Aerospace Exploration Agency (JAXA). Building upon the success of its predecessor, the Tropical Rainfall Measuring Mission (TRMM), GPM aims to unify and advance nextgeneration precipitation measurement from a constellation of both research and operational satellites (Hou et al. 2014). Launched on 28 February 2014, the GPM Core Observatory is equipped with the first spaceborne dualfrequency precipitation radar, and a conical-scanning multichannel microwave imager. Specifically, the GPM Microwave Imager (GMI) not only inherits the nine channels of the TRMM Microwave Imager (TMI) to detect heavy to light precipitation, but also includes four additional high-frequency channels $(166$ and $183 \mathrm{GHz})$ to improve sensitivity to and detection of snowfall. In addition, GMI at least doubles the spatial resolution of the channels in TMI and has one of the highest resolutions among the group of GPM constellation satellites. Furthermore, the outstanding calibration of GMI also serves as a reference for the intercalibration of other microwave sounders in the GPM constellation to ensure a physically consistent brightness temperature.

Because of the numerous improvements that GPM brings compared to TRMM, it is expected that assimilating GMI radiance will have a positive impact on hurricane track and intensity forecasts. In this study, we investigate the impact of assimilating GMI clear-sky radiance on hurricane track and intensity forecasts using the National Centers for Environmental Prediction (NCEP) Hurricane Weather Research and Forecasting (HWRF) Model and the Gridpoint Statistical Interpolation analysis system (GSI)-based hybrid of ensemble and three-dimensional variational (3DVar) data assimilation systems. Two notable Atlantic hurricane cases, Hurricanes Joaquin (2015) and Matthew (2016), are used for case studies in this paper.

The paper is organized as follows: section 2 gives a brief introduction to the GMI observations, HWRF Model, GSI data assimilation system, hurricane cases, and experimental setting designs. Section 3 provides details about quality control (QC) and bias correction (BC). Assimilation and forecast results and validation of the data impact are discussed in section 4. Section 5 summarizes results and provides the conclusions.

\section{GMI observations, HWRF Model, data assimilation system, and experimental configurations}

\section{a. GMI observations}

GMI is a conical scanning passive microwave radiometer with 13 microwave channels, ranging from 10 to $183 \mathrm{GHz}$. Channels 1-9 of GMI have frequencies $(10-89 \mathrm{GHz})$ similar to those of its predecessor, the TRMM Microwave Imager (TMI). Responsible for sensing liquid precipitation and the lower-troposphere moisture profile, these channels obtain moisture and temperature information in the lower troposphere $(>800 \mathrm{hPa})$. In addition, GMI includes four high-frequency channels (channels 10-13) that are responsible for detection of light precipitation and snowfall. Using a conical scan design, the GMI main reflector has a rotation rate of $32 \mathrm{rpm}$, scanning a $140^{\circ}$ sector centered on the spacecraft ground track with an altitude of about $407 \mathrm{~km}$. This scan configuration gives a cross-track swath covering roughly $885 \mathrm{~km}$ above the earth's surface. In terms of scan geometry, GMI uses two sets of scan angles for the 13 channels. The first 9 channels scan at an off-nadir angle of $48.5^{\circ}$, while the remaining 4 channels scan at $45.46^{\circ}$ (see details in Hou et al. 2014). In this study, the GMI Level 1C-R common calibrated and coregistered highfrequency and low-frequency brightness temperature data in all 13 microwave channels are assimilated. 


\section{b. HWRF Model, data assimilation system, and the forward model}

The HWRF system was developed at NCEP to provide numerical guidance for the forecasting of tropical cyclone tracks, intensity, and structure (Gopalakrishnan et al. 2011). It uses the Nonhydrostatic Mesoscale Model (NMM) core of the Weather Research and Forecasting (WRF) system as its dynamic solver. Since 2011, HWRF has adopted a triply nested domain configuration. Specifically, HWRF, version 3.7 [see details in Tallapragada et al. (2015)], is used in this study, in which the parent domain is configured with $18-\mathrm{km}$ horizontal resolution, covering roughly $80^{\circ} \times 80^{\circ}$ on a rotated latitudelongitude E-staggered grid. The intermediate and inner nest domains are two-way interactive nesting and move along with the storm, with resolutions of about 6 vand $2 \mathrm{~km}$, covering about $12^{\circ} \times 12^{\circ}$ and $7.1^{\circ} \times 7.1^{\circ}$, respectively (see Fig. 1). The configuration of the HWRF Model is set as close as possible to the operational HWRF Model in the hurricane season of 2015.

The current data assimilation system for HWRF is the NCEP GSI-based hybrid ensemble-3DVar system. The cost function used in the data assimilation system is defined as follows:

$$
\begin{aligned}
J(\mathbf{x})= & \frac{1}{2}\left(\mathbf{x}-\mathbf{x}^{b}\right)^{\mathrm{T}}\left(\beta_{1} \mathbf{B}_{1}+\beta_{2} \mathbf{B}_{2}\right)^{-1}\left(\mathbf{x}-\mathbf{x}^{b}\right) \\
& +\frac{1}{2}\left[\mathbf{y}_{0}-H(\mathbf{x})\right]^{\mathrm{T}} \mathbf{R}^{-1}\left[\mathbf{y}_{0}-H(\mathbf{x})\right],
\end{aligned}
$$

where the first term is the background error term with a hybrid background error covariance, which is a sum of two parts: a prescribed static matrix $\mathbf{B}_{1}$ and a flow-dependent part of the background covariance matrix $\mathbf{B}_{2}$ estimated using the 6-h forecast of the 80-member ensemble Kalman filter (EnKF) for the NCEP Global Forecast System (GFS). The weighting factors for $\left(\beta_{1}\right.$ and $\left.\beta_{2}\right)$ are set to be 0.2 and 0.8 , respectively. The second term is an observational error term. The operator $H$ is the forward model, which includes the Community Radiative Transfer Model (CRTM) as its radiative transfer component for satellite radiance assimilation. CRTM is a fast-radiative transfer model developed by the Joint Center for Satellite Data Assimilation (JCSDA) at NOAA. A comprehensive description of CRTM can be found in Han et al. (2006) and Weng (2007). CRTM is able to simulate radiances of a large number of sensors on board, including GMI. For clear-sky simulation over the ocean, basic inputs include atmospheric vertical profiles (e.g., temperature, specific humidity), surface parameters (e.g., surface temperature, wind speed, and direction) and sensor geometry (e.g., sensor zenith angle, sensor height). In addition to radiance computation, CRTM also includes calculation of the

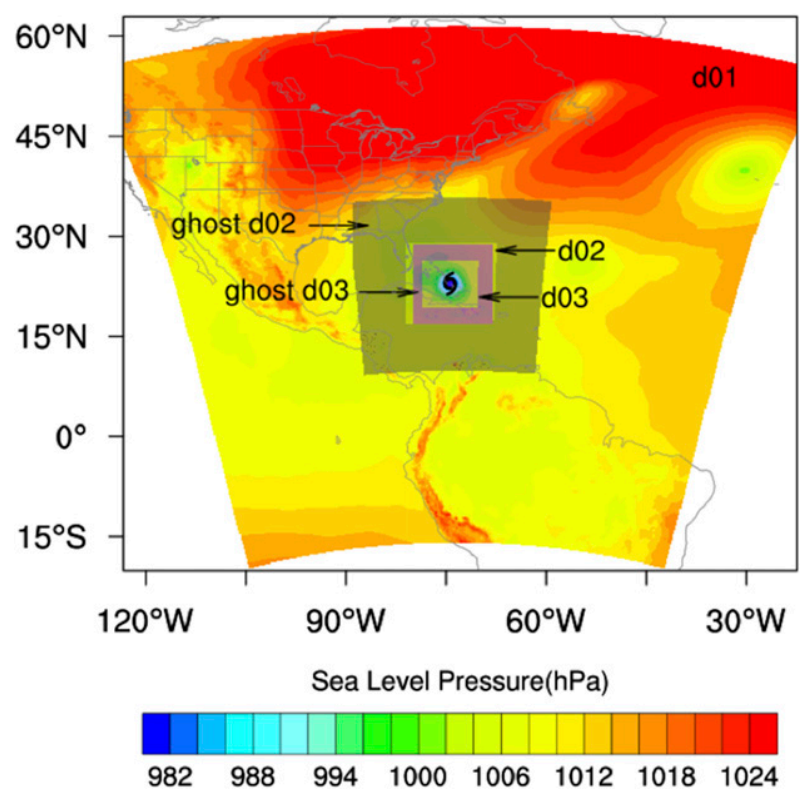

FIG. 1. GFS analysis of sea level pressure (color contours, units $\mathrm{hPa}$ ) and storm center from NHC best track (black storm sign) at 0000 UTC 2 Oct 2015 for Hurricane Joaquin. HWRF Model forecast domains, as indicated by $\mathrm{d} 01, \mathrm{~d} 02$, and d03 and HWRF data assimilation domains, as indicated by ghost $\mathrm{d} 02$ (black shaded area), and ghost d03 (pink shaded area) are also indicated.

Jacobian and adjoint model for facilitating various data assimilation-related applications.

\section{c. Experimental configurations}

In this study, two notable recent hurricanes cases, Hurricane Joaquin (2015) and Hurricane Matthew (2016), are selected in order to examine the impact of assimilation of GMI clear-sky radiance on hurricane track and intensity forecasts.

Before data assimilation, the HWRF Model employs a vortex initialization procedure (Liu et al. 2006) to correct the storm position and intensity approach to the real-time estimation (see details in Tallapragada et al. 2015). This procedure consists of two components: a vortex relocation, which places the hurricane vortex at the location from the National Hurricane Center (NHC) tropical cyclone vital statistics (TCVitals) database, and an intensity and size correction, which adjusts the intensity and vortex structure using the TCVitals database. However, previous studies have shown that vortex initialization and data assimilation counteract each other in some cases (Tallapragada et al. 2015). In addition, vortex initialization can also induce imbalances in the initial conditions and lead to vortex spindown problems in HWRF forecasts in some cases (Pu et al. 2016; Zhang et al. 2018). In these situations, impacts from data assimilation can be hard to interpret clearly. 
To clearly demonstrate the impact of GMI data assimilation, the intensity correction component is turned off in all data assimilation experiments presented in this study. However, the vortex relocation is still used for data assimilation experiments to avoid potential phase errors that may put observations in wrong locations and result in harm to the vortex inner core.

In addition, the current operational HWRF uses the NCEP GFS analysis to initialize the outer domain (d01) and also uses the GFS forecast to provide the boundary conditions for d01. Since observations over the parent domain are already assimilated in the global data assimilation, the operational HWRF data assimilation is performed only at the inner 6- and 2-km nests (Biswas et al. 2015). However, since GMI radiance has not yet been assimilated in the operational GFS, assimilation of GMI radiance over the parent domain is desired in order to assess its full data impact. To achieve this, we initialize the parent domain using the GFS 6-h forecast, employing the same procedure as in the inner 6- and 2-km inner nests to achieve data assimilation over the parent domain. In each of these experiments, the parent domain assimilates the same types of data as are assimilated in the ghost domain 2.

Several sets of data assimilation experiments (with and without GMI data) are performed to evaluate the impact of assimilating GMI radiance on hurricane simulations (see the list of experiments in Table 1) using the GSI-based ensemble-3DVar (hereafter GSI for simplicity) data assimilation system. Each set of experiments consists of a control experiment (CTRL), which assimilates all data (except for the tail Doppler radar data because the data were absent in many cases in this study) that are currently assimilated in operational HWRF, including conventional data, GPS radio occultation (RO) data, satellite-derived winds, and satellite radiance observations from both microwave [e.g., Advanced Microwave Sounding Unit-A (AMSU-A), Advanced Technology Microwave Sounder (ATMS), Microwave Humidity Sounder (MHS), etc.] and infrared [e.g., Atmospheric Infrared Sounder (AIRS), Infrared Atmospheric Sounding Interferometer (IASI), etc.] sensors, and the GMI data assimilation experiment (GMI), which is the same as CTRL but with GMI clearsky radiance assimilated in the parent domain and ghost domain 2. For all experiments, the HWRF Model is spun up by regular 6-hourly analysis-forecast cycles with all the data assimilated into the CTRL experiments (as described above) till $6 \mathrm{~h}$ before the data assimilation experiments with GMI. Specifically, for Hurricane Joaquin, the mature phase of its life cycle is emphasized for the experiments in this study. The spinup period is from 0000 UTC 29 September 2015 to 0000 UTC 1 October 2015. Then, the data assimilation experiments are performed in 6-hourly analysis-forecast cycles from 0600 UTC 1 October to 1800 UTC 2 October 2015 with GMI data, with the configurations of CTRL1 and GMI1 in Table 1. For Hurricane Matthew, both the genesis and mature phases are included. For its genesis phase, the spinup period is from 1200 UTC 26 September (when Matthew was still a tropical disturbance) to 1200 UTC 27 September 2016, and the data assimilation experiments (CTRL2 and GMI 2 in Table 1) are performed in 6-hourly analysis-forecast cycles from 1800 UTC 27 September to 0000 UTC 29 September 2016. For its mature phase, the HWRF Model is spun up from 1800 UTC 30 September 2016 to 1800 UTC 2 October 2016, and the data assimilation experiments (CTRL3 and GMI3 in Table 1) are performed from 0000 UTC 3 October to 1200 UTC 4 October 2016. The 120-h forecasts are performed at each analysis time after the data assimilation.

\section{GMI data quality control and bias correction}

\section{a. Quality control $(Q C)$}

The QC for GMI clear-sky radiance in the GSI data assimilation system uses two parameters associated with cloud liquid water (CLW) and cloud ice, and three parameters associated with surface emissivity (following Garrett et al. 2010).

First, over the sea surface, a column CLW is computed using measurements from GMI channels $1-7,12$, and 13:

$$
\begin{aligned}
\mathrm{CLW}= & a_{0, \mathrm{clw}}+\sum_{i=1-7,} c_{i} T_{b, i}+\hat{c}_{1} \log \left(T_{b, 3}-T_{b, 4}\right) \\
& +\hat{c}_{2} \log \left(T_{b, 6}-T_{b, 7}\right),
\end{aligned}
$$

where $c_{i}(i=1-7,12,13)$ and $\hat{c}_{1}$ and $\hat{c}_{2}$ are prescribed regression coefficients, and $T_{b, i}(i=1-7,12,13)$ is the GMI brightness temperature at channel $i$. Another column cloud ice [e.g., graupel water path (GWP)] parameter is computed in a similar fashion:

$$
\mathrm{GWP}=a_{0, \mathrm{gwp}}+\sum_{\substack{i=1-7, 12,13}} g_{i} T_{b, i}+\hat{g}\left[300-\log \left(T_{b, 10}\right)\right] .
$$

Table 2 summarizes the values of $c_{i}, \hat{c}_{i}, g_{i}$, and $\hat{g}$. Constant thresholds of 0.05 are used for both CLW and GWP to toss pixels that are considered to be contaminated by CLW emission and ice scattering. 
TABLE 1 . The list of experiment configurations.

\begin{tabular}{|c|c|c|c|c|c|}
\hline Expt & $\begin{array}{l}\text { Hurricane } \\
\text { case }\end{array}$ & $\begin{array}{l}\text { Data assimilation } \\
\text { (DA) period }\end{array}$ & DA domains and data & $\begin{array}{c}\text { DA cycle in d01 } \\
\text { with GMI overpass }\end{array}$ & $\begin{array}{c}\text { DA cycle in d02 } \\
\text { with GMI overpass }\end{array}$ \\
\hline CTRL1 & $\begin{array}{l}\text { Joaquin }(2015) \\
\text { mature }\end{array}$ & $\begin{array}{l}0600 \text { UTC } 1 \\
\text { Oct-1800 } \\
\text { UTC } 2 \text { Oct }\end{array}$ & $\begin{array}{l}\text { d01: Conventional observations, } \\
\text { satellite observations } \\
\text { d02: Conventional observations, } \\
\text { satellite observations } \\
\text { d03: Conventional observations }\end{array}$ & & \\
\hline \multirow[t]{4}{*}{ GMI1 } & $\begin{array}{l}\text { Joaquin }(2015) \\
\text { mature }\end{array}$ & $\begin{array}{r}0600 \text { UTC } 1 \\
\text { Oct-1800 }\end{array}$ & $\begin{array}{l}\text { d01: Conventional observations, } \\
\text { satellite observations, GMI }\end{array}$ & 0600 UTC 1 Oct 2015 & 0600 UTC 1 Oct 2015 \\
\hline & & UTC 2 Oct & $\begin{array}{l}\text { d02: Conventional observations, } \\
\text { satellite observations, GMI }\end{array}$ & 1200 UTC 1 Oct 2015 & 0000 UTC 2 Oct 2015 \\
\hline & & & d03: Conventional observations & $\begin{array}{l}1800 \text { UTC } 1 \text { Oct } 2015 \\
0000 \text { UTC } 2 \text { Oct } 2015\end{array}$ & 0600 UTC 2 Oct 2015 \\
\hline & & & & $\begin{array}{l}0600 \text { UTC } 2 \text { Oct } 2015 \\
1800 \text { UTC } 2 \text { Oct } 2015\end{array}$ & 1800 UTC 2 Oct 2015 \\
\hline CTRL2 & $\begin{array}{l}\text { Matthew (2016) } \\
\text { genesis }\end{array}$ & $\begin{array}{l}1800 \text { UTC } 27 \\
\text { Sep-0000 } \\
\text { UTC } 29 \text { Sep }\end{array}$ & $\begin{array}{l}\text { d01: Conventional observations, } \\
\text { satellite observations } \\
\text { d02: Conventional observations, } \\
\text { satellite observations } \\
\text { d03: Conventional observations }\end{array}$ & & \\
\hline \multirow[t]{5}{*}{ GMI2 } & $\begin{array}{l}\text { Matthew (2016) } \\
\text { genesis }\end{array}$ & $\begin{array}{l}1800 \text { UTC } 27 \\
\text { Sep-0000 }\end{array}$ & $\begin{array}{l}\text { d01: Conventional observations, } \\
\text { satellite observations, GMI }\end{array}$ & 1800 UTC 27 Sep 2016 & 1800 UTC 27 Sep 2016 \\
\hline & & UTC 29 Sep & $\begin{array}{l}\text { d02: Conventional observations, } \\
\text { satellite observations, GMI }\end{array}$ & 0600 UTC 28 Sep 2016 & 1200 UTC 28 Sep 2016 \\
\hline & & & d03: Conventional observations & 1200 UTC 28 Sep 2016 & 0000 UTC 29 Sep 2016 \\
\hline & & & & 1800 UTC 28 Sep 2016 & \\
\hline & & & & 0000 UTC 29 Sep 2016 & \\
\hline \multirow[t]{2}{*}{ CTRL3 } & $\begin{array}{l}\text { Matthew (2016) } \\
\text { mature }\end{array}$ & $\begin{array}{c}0000 \text { UTC } 3 \\
\text { Oct-1200 }\end{array}$ & $\begin{array}{l}\text { d01: Conventional observations, } \\
\text { satellite observations }\end{array}$ & & \\
\hline & & UTC 4 Oct & $\begin{array}{l}\text { d02: Conventional observations, } \\
\text { satellite observations } \\
\text { d03: Conventional observations }\end{array}$ & & \\
\hline \multirow[t]{7}{*}{ GMI3 } & $\begin{array}{l}\text { Matthew (2016) } \\
\text { mature }\end{array}$ & $\begin{array}{c}0000 \text { UTC } 3 \\
\text { Oct }-1200\end{array}$ & $\begin{array}{l}\text { d01: Conventional observations, } \\
\text { satellite observations, GMI }\end{array}$ & 0000 UTC 3 Oct 2016 & 0000 UTC 3 Oct 2016 \\
\hline & & UTC 4 Oct & $\begin{array}{l}\text { d02: Conventional observations, } \\
\text { satellite observations, GMI }\end{array}$ & 0600 UTC 3 Oct 2016 & 0600 UTC 3 Oct 2016 \\
\hline & & & d03: Conventional observations & 1200 UTC 3 Oct 2016 & 1200 UTC 3 Oct 2016 \\
\hline & & & & 1800 UTC 3 Oct 2016 & 1800 UTC 3 Oct 2016 \\
\hline & & & & 0000 UTC 4 Oct 2016 & 1200 UTC 4 Oct 2016 \\
\hline & & & & 0600 UTC 4 Oct 2016 & \\
\hline & & & & 1200 UTC 4 Oct 2016 & \\
\hline \multirow[t]{2}{*}{ Base } & $\begin{array}{l}\text { Matthew (2016) } \\
\text { genesis }\end{array}$ & $\begin{array}{l}1800 \text { UTC } 27 \\
\text { Sep-0000 }\end{array}$ & $\begin{array}{l}\text { d01: Conventional observations, } \\
\text { no satellite observations }\end{array}$ & & \\
\hline & & UTC 29 Sep & $\begin{array}{l}\text { d02: Conventional observations, } \\
\text { no satellite observations } \\
\text { d03: Conventional observations }\end{array}$ & & \\
\hline \multirow[t]{5}{*}{ Base + GMI } & $\begin{array}{l}\text { Matthew (2016) } \\
\text { genesis }\end{array}$ & $\begin{array}{l}1800 \text { UTC } 27 \\
\text { Sep-0000 }\end{array}$ & $\begin{array}{l}\text { d01: Conventional observations, } \\
\text { add GMI }\end{array}$ & 1800 UTC 27 Sep 2016 & 1800 UTC 27 Sep 2016 \\
\hline & & UTC 29 Sep & $\begin{array}{l}\text { d02: Conventional observations, } \\
\text { add GMI }\end{array}$ & 0600 UTC 28 Sep 2016 & 1200 UTC 28 Sep 2016 \\
\hline & & & d03: Conventional observations & 1200 UTC 28 Sep 2016 & 0000 UTC 29 Sep 2016 \\
\hline & & & & 1800 UTC 28 Sep 2016 & \\
\hline & & & & 0000 UTC 29 Sep 2016 & \\
\hline
\end{tabular}

In addition, the GSI surface emissivity QC filters pixels affected by errors in the modeling of surface emissivity using channels that are less susceptible to the emission and absorption of water vapor (e.g., 10.65, 18.87 , and $36.5 \mathrm{GHz}$, each of which contributes one emissivity parameter). The emissivity parameters for these channels are calculated from an emissivity regression using all 13 GMI channel $T_{b}$ values and the model surface skin temperature. A pixel is considered to be affected by surface emissivity error if any of these 
TABLE 2. Prescribed regression coefficients to calculate CLW and GWP for GMI brightness temperature included in GSI, version 3.3 (after Garrett et al. 2010).

\begin{tabular}{crcr}
\hline \hline & CLW & \multicolumn{2}{c}{ GWP } \\
\hline$a_{0, \text { clw }}$ & -0.61127 & $a_{0, \text { gwp }}$ & -3541.46329 \\
$c_{1}$ & 0.00378 & $g_{1}$ & 0.00393 \\
$c_{2}$ & -0.00149 & $g_{2}$ & 0.00088 \\
$c_{3}$ & 0.03438 & $g_{3}$ & 0.00063 \\
$c_{4}$ & 0.01670 & $g_{4}$ & -0.00683 \\
$c_{5}$ & 0.00228 & $g_{5}$ & 0.00333 \\
$c_{6}$ & 0.03884 & $g_{6}$ & -0.00382 \\
$c_{7}$ & 0.02345 & $g_{7}$ & 0.00452 \\
$c_{12}$ & -0.00036 & $g_{12}$ & 0.04765 \\
$c_{13}$ & 0.00044 & $g_{13}$ & -0.00491 \\
$\hat{c}_{1}$ & 1.95559 & $\hat{g}$ & 11.98897 \\
$\hat{c}_{2}$ & 2.15143 & & \\
\hline
\end{tabular}

three parameters exceeds a corresponding prescribed threshold. Figure 2 shows a sample of the spatial distribution of data from GMI channel-5 brightness temperatures that pass and fail the QC in the GSI system.

\section{b. Bias correction $(B C)$}

All data assimilation systems are developed based on the assumption of unbiased Gaussian observational errors. However, for satellite radiance observations, sources of bias can arise from instrument error, such as calibration error and degradation of the instrument over time, systematic modeling error embedded in the radiative transfer model (including the implicit assumptions of the absence of cloud liquid and cloud ice), and variation of the scan angle that causes changes in the field of view at different scan positions. While random error can be handled by the data assimilation system, systematic errors (biases) can ultimately degrade the analysis, as the data assimilation system implicitly assumes an unbiased observational error (Dee 2004; Migliorini 2012). Therefore, systematic error has to be removed from the observations before passing them to the data assimilation system.

Bias correction for satellite observations consists of two main components: airmass-dependent bias and scan angle-dependent bias. Since GSI, version 3.3, bias correction for these two components has been performed in a single step in the enhanced radiance bias correction framework. Specifically, the airmass-dependent bias for each GMI channel $j$ is projected by a weighted linear sum of a static bias term $b_{j}$, airmass-dependent predictors $\left\{p_{i, j}^{(1)}\right\}$, and scan angle $\left\{p_{i, j}^{(2)}\right\}$ :

$$
b_{j}=b_{0, j}+\sum_{i=1}^{3} \beta_{i, j} p_{i, j}^{(1)}(x)+\sum_{l=1}^{4} \alpha_{l, j} \phi^{i},
$$

where $\beta_{i, j}(i=1,2,3)$ and $\alpha_{l, j}(l=1, \ldots, 4)$ are bias correction coefficients for airmass components and scan angle components, respectively; and $\phi$ is the field of view number (FOVN). The airmass-dependent predictor $p_{i, j}^{(1)}(i=1,2,3)$ is defined as follows:

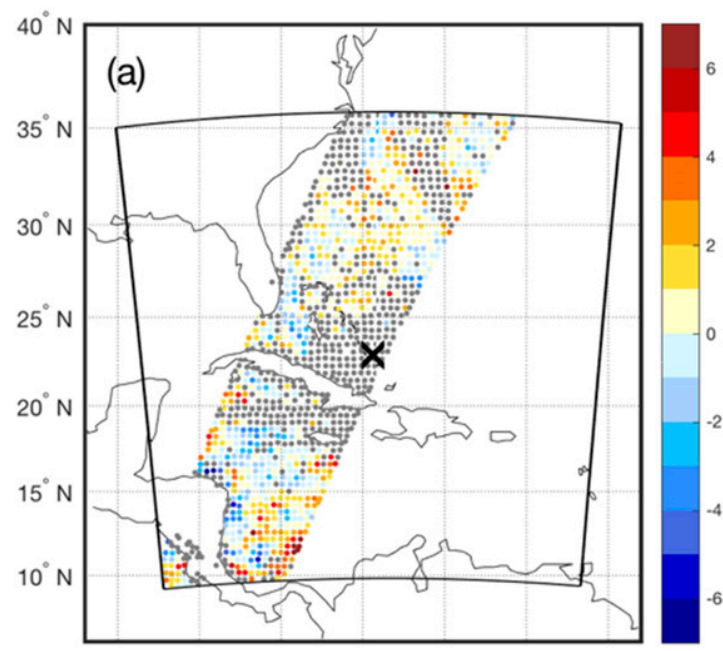

$90^{\circ} \mathrm{W} 85^{\circ} \mathrm{W} 80^{\circ} \mathrm{W} 75^{\circ} \mathrm{W} 70^{\circ} \mathrm{W} 65^{\circ} \mathrm{W} \quad 60^{\circ} \mathrm{W}$

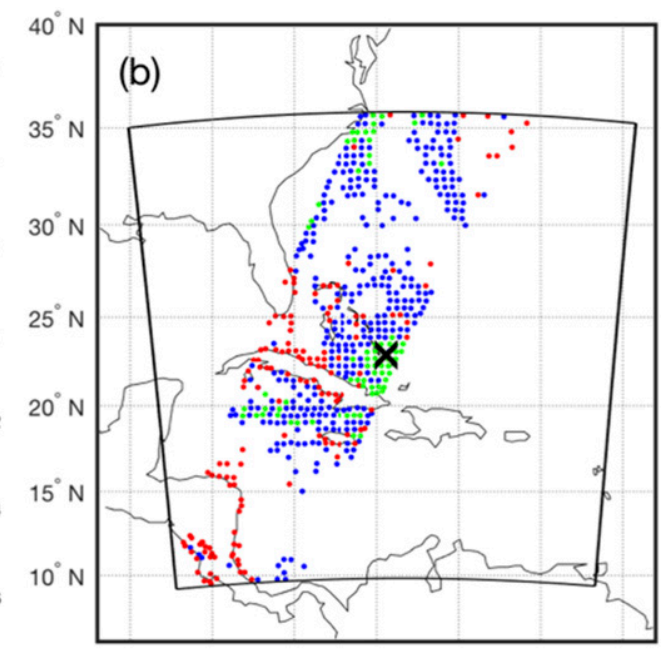

$90^{\circ} \mathrm{W} 85^{\circ} \mathrm{W} 80^{\circ} \mathrm{W} 75^{\circ} \mathrm{W} 70^{\circ} \mathrm{W} 65^{\circ} \mathrm{W} 60^{\circ} \mathrm{W}$

FIG. 2. Spatial distribution of (a) GMI overpass over ghost d02 at 0000 UTC 2 Oct 2015, with data that passed QC (colored with $O-F$ values) and failed QC (gray), and (b) GMI observations that failed QC shaded with different colors to denote the type of failure (blue: CLW QC; green: GWP QC; red: emissivity QC). Ghost d02 is shown as an inner domain inside a longitude-latitude box. The hurricane center is indicated by the black cross at the domain center. 

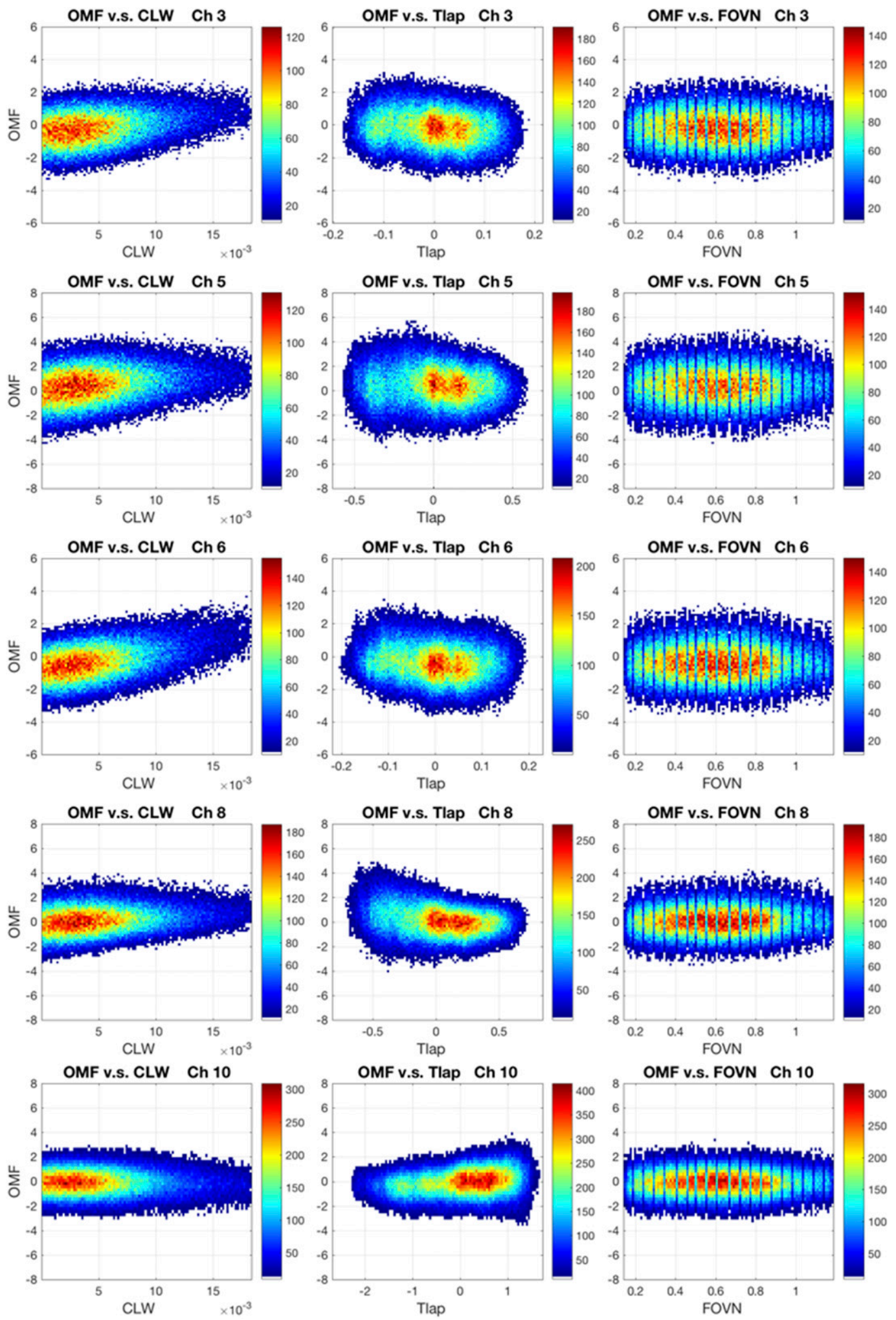

FIG. 3. Two-dimensional histogram showing the dependence of $O-F$ sample (collected from 26 Sep to 9 Oct 2015) on (left) CLW $\left(\mathrm{kg} \mathrm{m}^{-2}\right)$, (middle) Tlap (K), and (right) the field of view number (FOVN; rad) for channels 3 , $5,6,8$, and 10 . The $y$ axis denotes $O-F$ value and the $x$ axis represents predictor. The flat distribution around 0 is unbiased. 
(a)

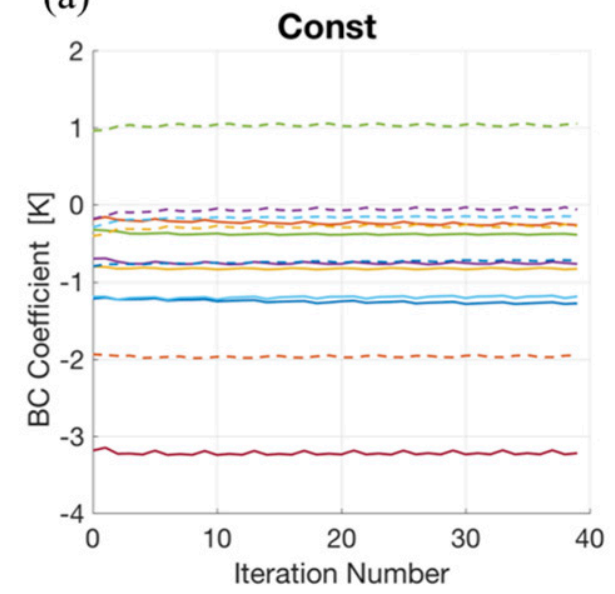

(c)

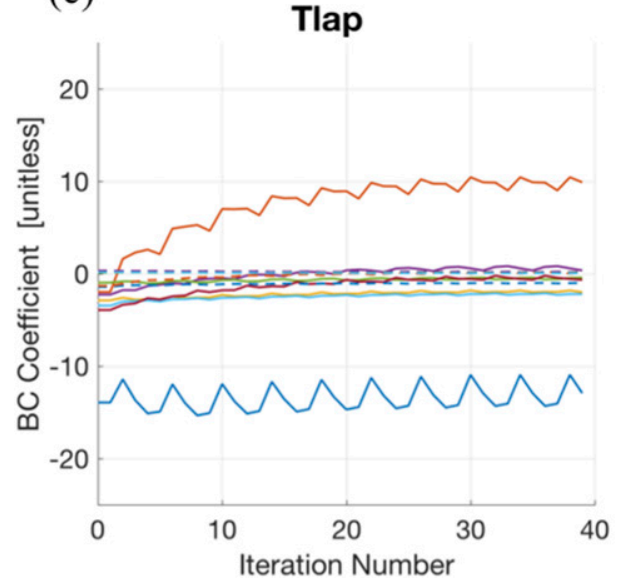

(b)

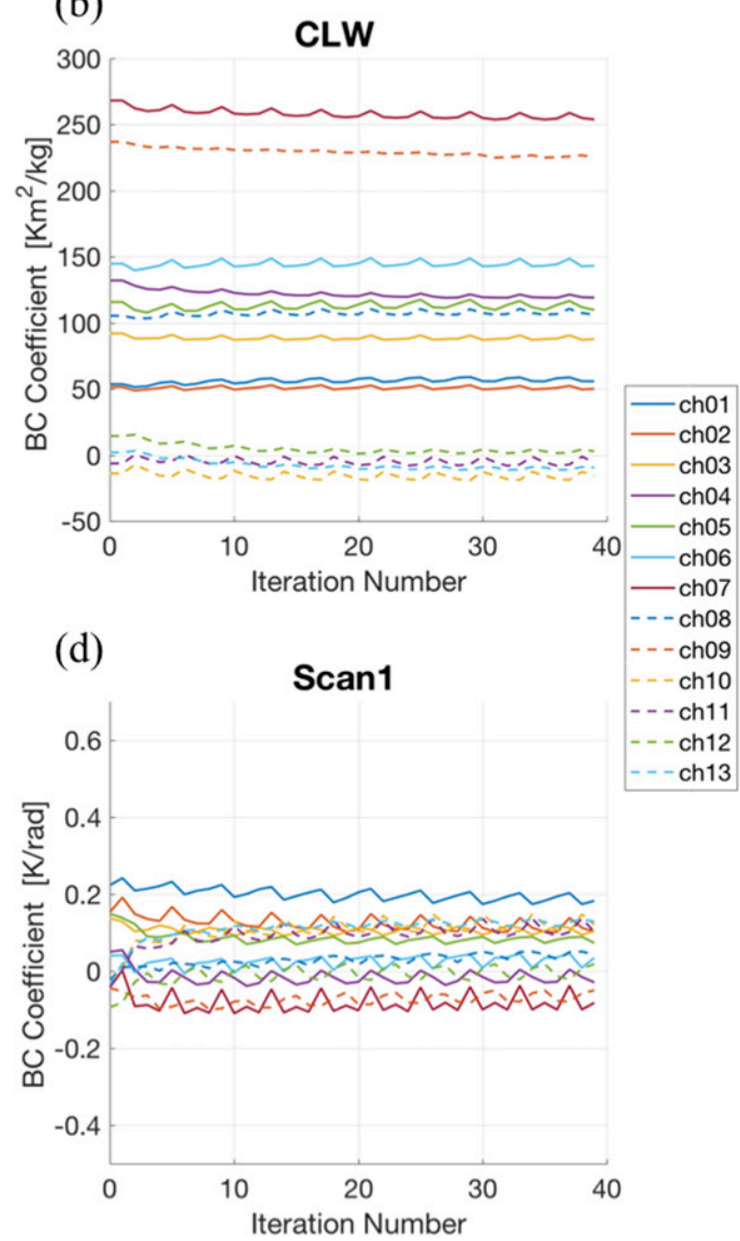

FIG. 4. Variation of BC coefficients with the iteration number obtained from experiment GMI1 for (a) constant offset, (b) CLW predictor (CLW), (c) transmittance lapse rate (Tlap), and (d) Scan angle.

$$
\begin{aligned}
& p_{1, j}^{(1)}=[\cos (\phi)]^{2} \times \mathrm{CLW}, \\
& p_{2, j}^{(1)}=\left(\Gamma_{j}^{\tau}-\bar{\Gamma}_{j}^{\tau}\right)^{2}, \\
& p_{3, j}^{(1)}=\Gamma_{j}^{\tau}-\bar{\Gamma}_{j}^{\tau},
\end{aligned}
$$

where $\Gamma_{j}^{\tau}$ is the lapse rate of transmittance for channel $j$ and varies over pixels, and $\bar{\Gamma}_{j}^{\tau}$ is the mean lapse rate of temperature. The $\Gamma_{j}^{\tau}$ in (6) and (7) is computed by the following expression:

$$
\Gamma_{j}^{\tau}=-\sum_{k=1}^{K}\left(\tau_{j, k+1}-\tau_{j, k}\right)\left(T_{k+1}-T_{k-1}\right),
$$

where $\tau_{j, k}$ is the transmittance from the $k$ th model level to the top of atmosphere for the $j$ th channel and is computed during CRTM integration, while $T_{k}$ is the temperature at the $k$ th model level.

\section{c. BC coefficients for GMI clear-sky radiances}

In operational HWRF, bias correction coefficients for each sensor are prescribed in the input GFS files. However, since GMI radiance data had not yet been assimilated in the operational GFS in 2015 and 2016, BC coefficients for GMI radiance observations were not available in these input data. Therefore, in this study, the BC coefficients for GMI radiances are derived independently using a combination of a static BC method and the adaptive BC capability within GSI. Specifically, a rough estimation of the $\mathrm{BC}$ coefficients is computed using a linear regression on a representative set of observations minus forecasts $(O-F)$ derived from 14 days of GMI overpasses in the region of interest. This rough estimation of coefficients is then treated as an initial guess for a variational BC inside of GSI through an iteration process over a short period of data assimilation cycles 

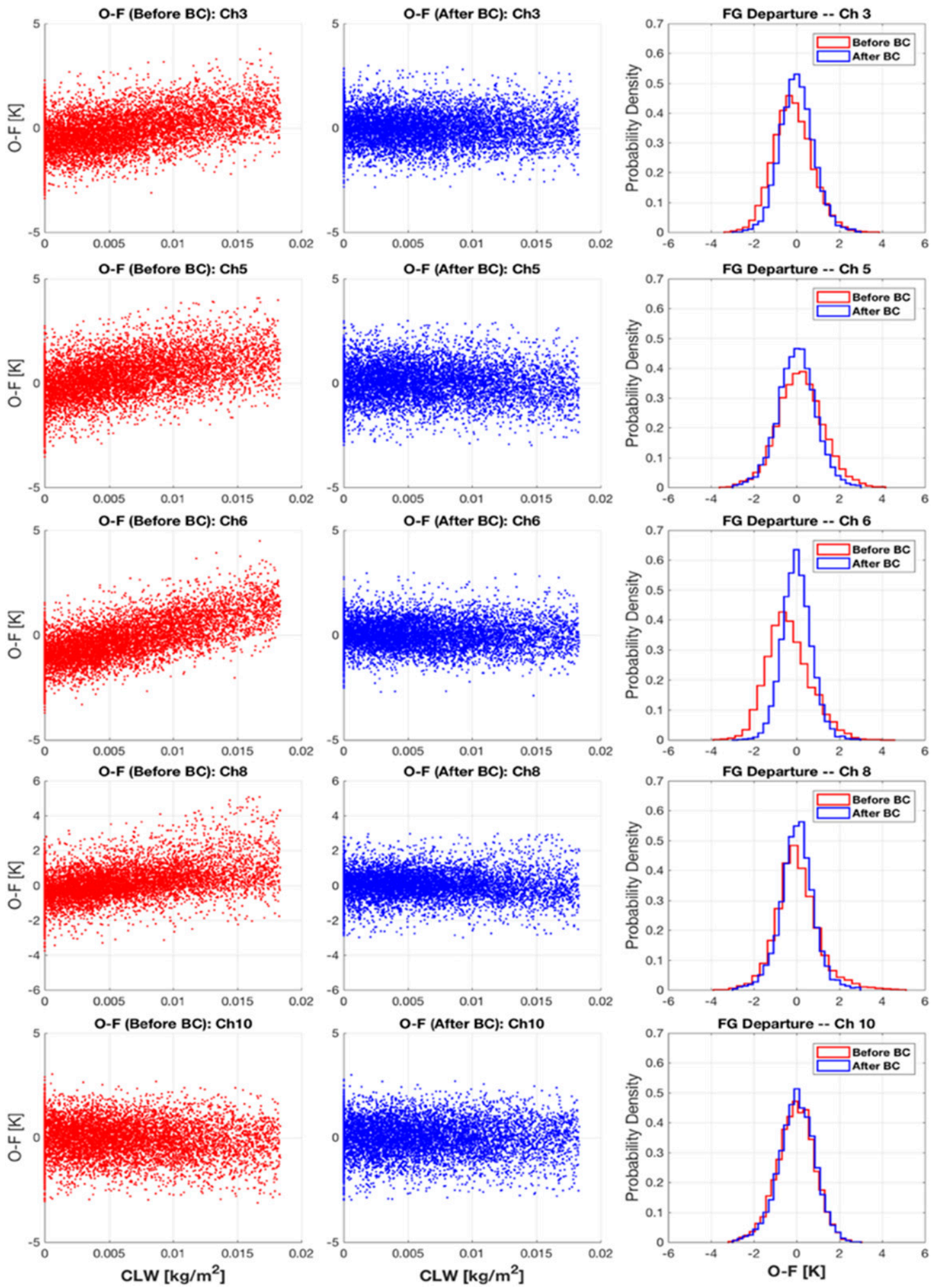

FIG. 5. $O-F$ statistics against CLW predictor (left) before and (middle) after BC. (right) Histogram of $O-F$ values. Data sample consists of assimilated GMI observations from four analysis-forecast cycles of the mature phase of Joaquin that have major GMI overpasses in the parent domain (0600 UTC 1 Oct, 1800 UTC 1 Oct, 0200 UTC 2 Oct, and 0600 UTC 2 Oct 2015). 

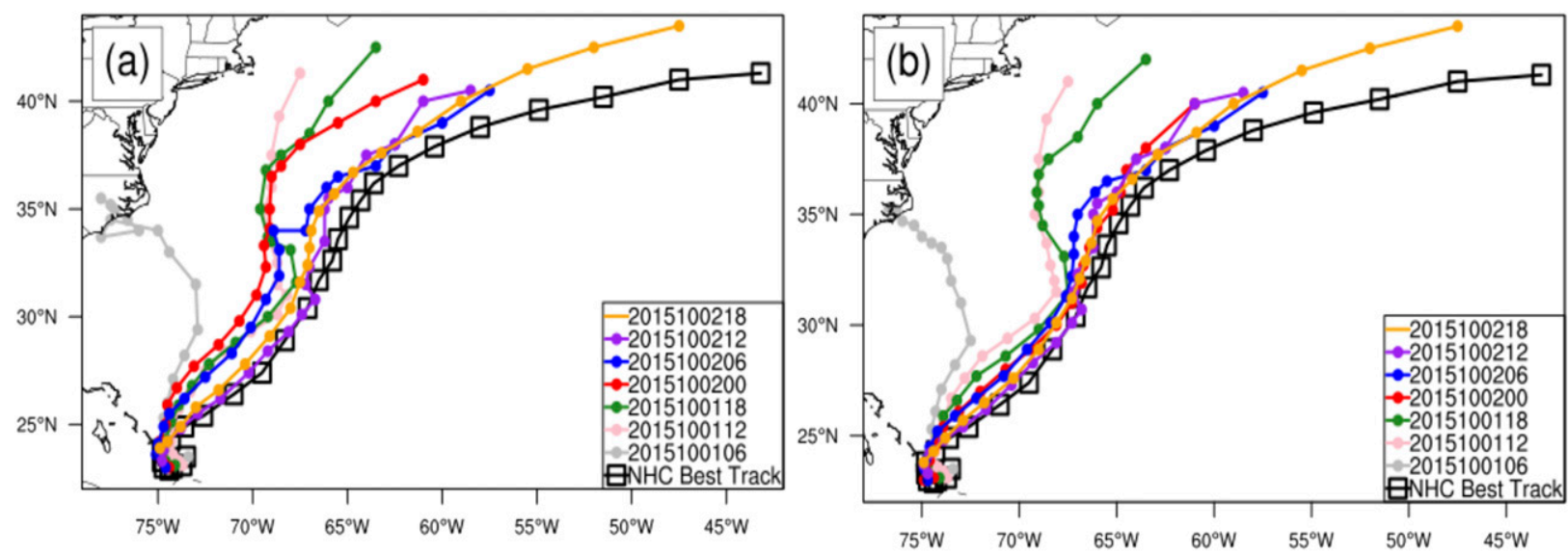

(c) CTRL1 vs. GMl1

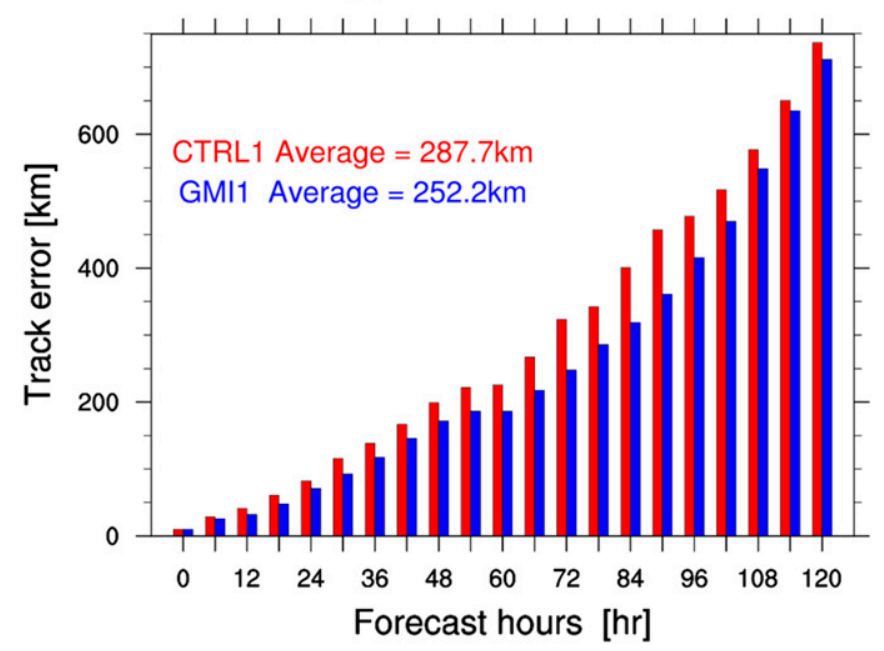

FIG. 6. Comparison of tracks between NHC best track (black curve) with 120-h HWRF forecasts (colored lines) initialized by each of the analyses generated in the 7 analysis-forecast cycles (as listed in the legend) for experiments without and with GMI data assimilation for (a) CTRL1 and (b) GMI1. (c) The 120-h mean track error averaged over the all 7 forecasts in (a) and (b), for CTRL1 (red) and GMI1 (blue).

(e.g., following Zhu et al. 2014) to obtain the temporal variation of the coefficients.

First, for each hurricane case, a set of 14-day overpass GMI data over the corresponding basin (North Atlantic Ocean for Hurricanes Joaquin and Matthew) is collected, which spans from the genesis phase to the decay phase of the hurricane. Meanwhile, for each GMI overpass, the departure between the simulated and observed $T_{b}$ (e.g., observed - first guess, or $O-F$ ) and the predictors [e.g., CLW, temperature lapse rate (Tlap) and its square, four orders of scan angles, mean bias or constant offsets] for each pixel are calculated. A multilinear regression is then used to estimate the dependence of $O-F$ values on a set of predictors and the $\mathrm{BC}$ coefficients are computed. Figure 3 shows sample results of the dependence of $O-F$ on predictors based on statistics during the life of Hurricane
Joaquin (between 26 September and 9 October 2015) for channels $3,5,6,8$, and 10 . There is apparent dependence between $O-F$ and the CLW and temperature lapse rate (Tlap), respectively, with biases present, but there is no dependence between $O-F$ and the scan angles.

As mentioned, the current version of GSI uses variational bias correction to adaptively update the $\mathrm{BC}$ coefficients by including the coefficients as analysis variables in the data assimilation step. This advancement allows the data assimilation system to adjust the BC coefficients automatically at each analysis step and capture the temporal evolution of the coefficients effectively (Zhu et al. 2014). Using this capability of the GSI system, a GSI iteration experiment is performed to finetune the $\mathrm{BC}$ coefficients for different phases of the hurricane case studies. Details of the iteration 


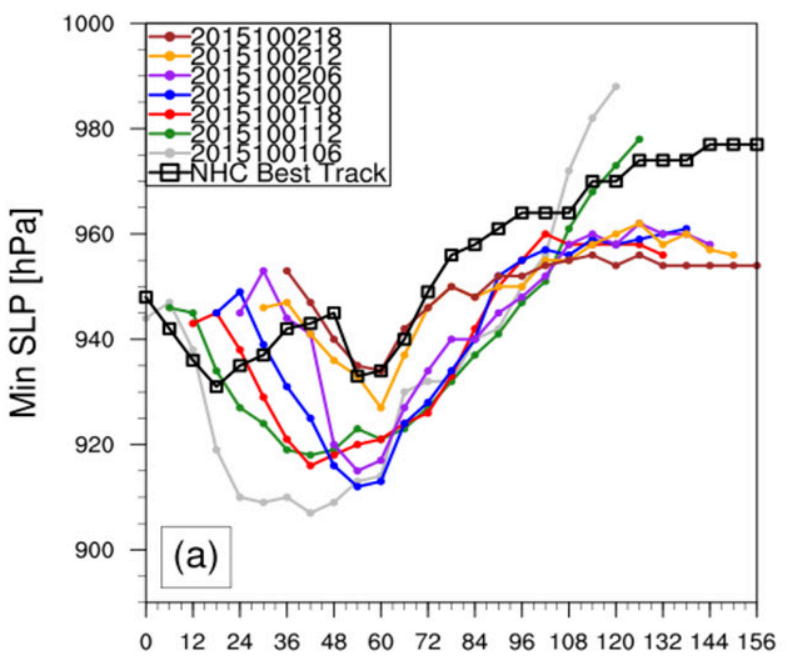

Hours since 06 UTC 01 Oct 2015

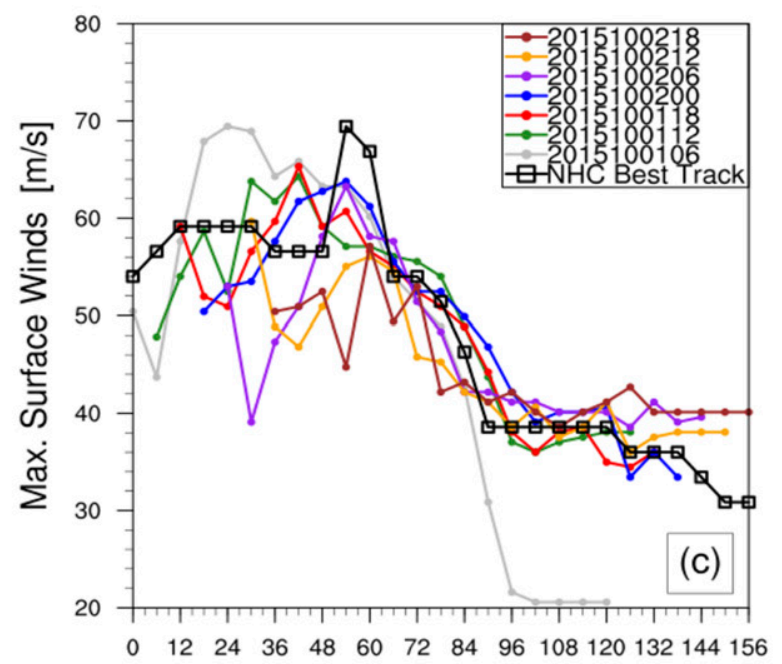

Hours since 06 UTC 01 Oct 2015

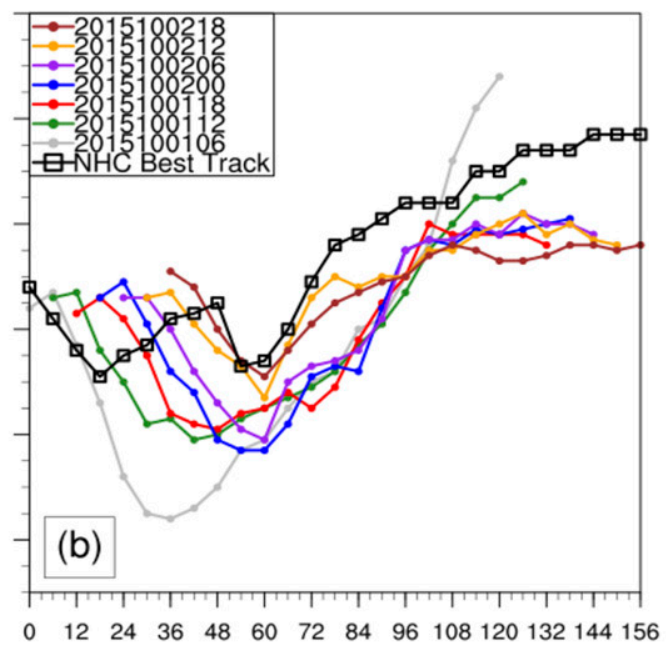

Hours since 06 UTC 01 Oct 2015

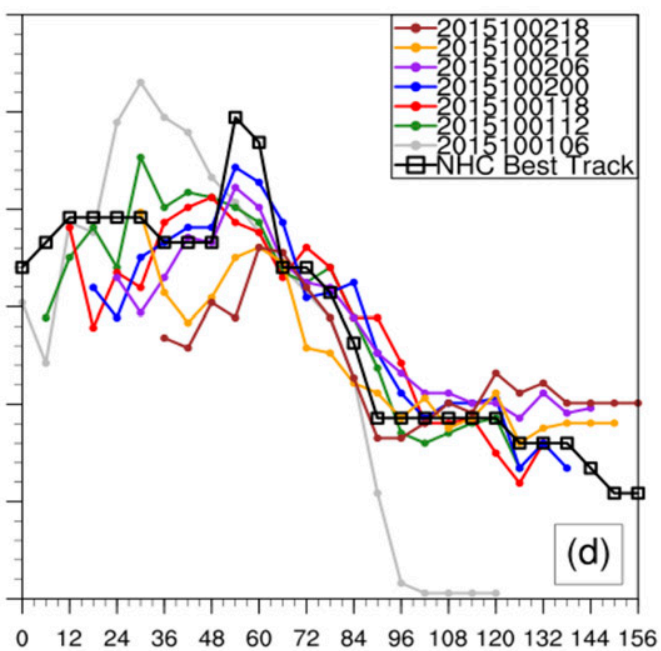

Hours since 06 UTC 01 Oct 2015


FIG. 7. Aggregated minimum SLP and maximum surface wind forecasts of (a),(c) CTRL1 and (b),(d) GMI1 (colored curves) compared with NHC best track data (black curve; 0600 UTC 1 Oct to 1800 UTC 7 Oct 2015) for the 120-h forecast started at the analysis time in all 7 analysis-forecast cycles (as listed in the legend). (e),(f) The mean forecast errors of minimum sea level pressure and maximum surface wind averaged over the forecasts started at the analysis time in all 6 analysis-forecast cycles (6-hourly from 0600 UTC 1 Oct to 1800 UTC 2 Oct 2015) for CTRL1 (red) and GMI1 (blue). In (e) and (f), the colored numbers on the left and right show the averaged errors over all 7 forecasts in first 60-h and 120-h HWRF integration, respectively. 

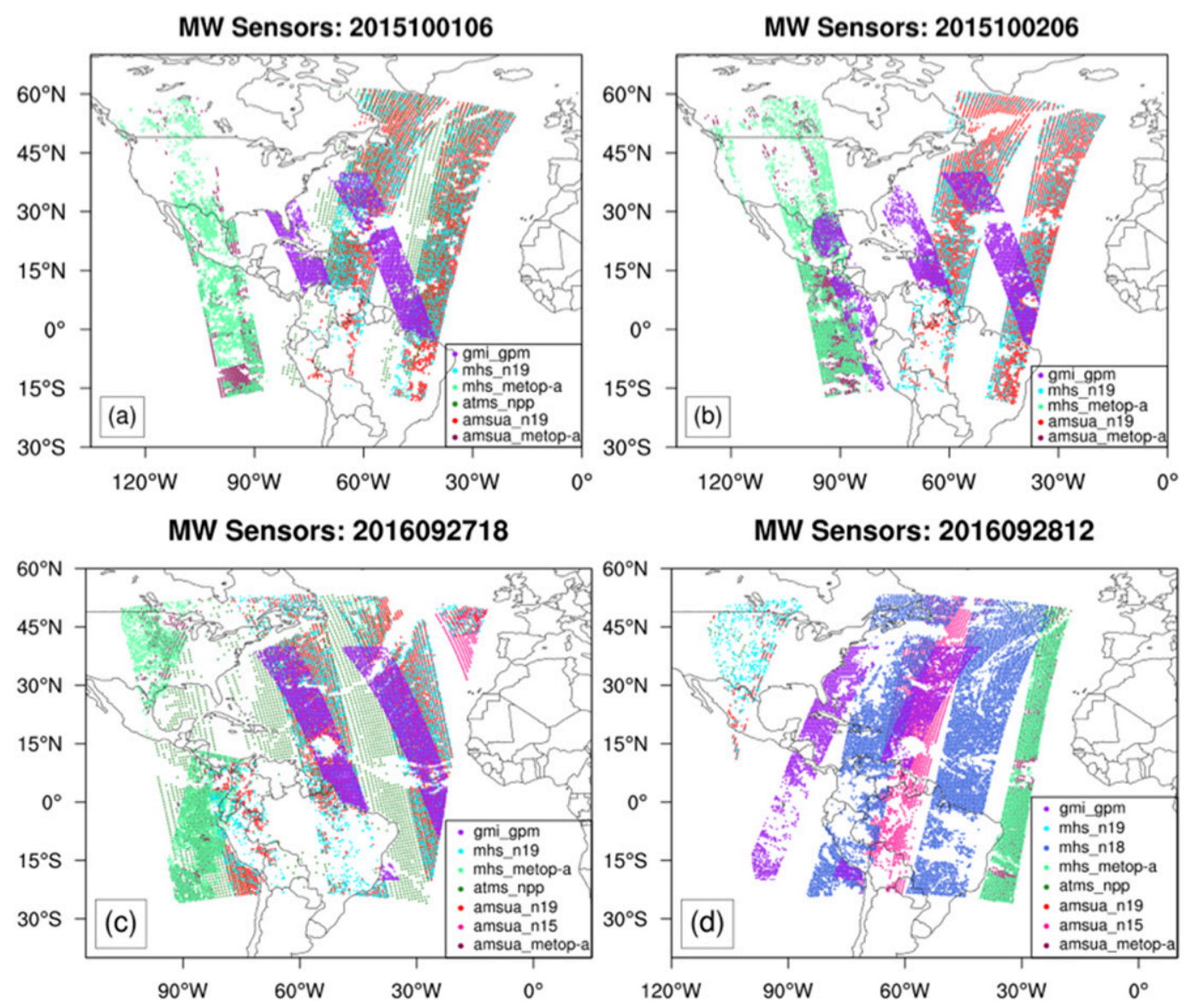

FIG. 8. Assimilated brightness temperature observations of all microwave sensors during the mature phase of Joaquin at (a) 0600 UTC 1 Oct and (b) 0600 UTC 2 Oct 2015, and during analysis cycles of the mature phase of Matthew at (c) 1800 UTC 27 Sep and (d) 1200 UTC 28 Sep 2016. GMI overpasses are highlighted in purple.

procedure are as follows: first, for a given hurricane (e.g., Joaquin), at least four 6-hourly data assimilation analysis-forecast cycles (each of which has at least one GMI overpass near the hurricane region) are performed. Then, using the BC coefficients obtained from linear regression as an initial guess, GSI analysis is performed sequentially at each of these 6-hourly analysis-forecast cycles. At each GSI analysis, the BC coefficients are adjusted accordingly and then passed to the next analysis-forecast cycle. After the coefficients go through all data assimilation within the analysis window, one iteration is completed and the whole process is repeated again for the next iteration until the coefficients stabilize. Figure 4 shows the variation of various predictors during the iteration procedure. It shows that the BC coefficients are stable after only several iterations for most variables. In fact, if the linear regression step is eliminated and the variational bias correction alone is used, the bias coefficients will converge (or become stable) slowly and can take more than 50 iterations to reach similar results. Zhu et al. (2014) have noted that poor initial guesses of BC coefficients may lead the QC to reject/accept data of good/poor quality.

Figure 5 summarizes the $O-F$ statistics before and after bias correction. The data sample consists of assimilated GMI observations from the first four analysisforecast cycles in the GMI data assimilation experiment during the mature phase of Joaquin in HWRF d01. The comparison shows that before $\mathrm{BC}, O-F$ dependence on CLW follows the same trend shown in the left column of Fig. 3. After BC, $O-F$ values are distributed almost uniformly over the entire range of CLW values. This indicates that the $\mathrm{BC}$ coefficients obtained from the twostep bias correction approach are able to remove bias arising from CLW emission. In addition, the right column of Fig. 5 shows a histogram of the first-guess departure before and after BC, revealing that after BC, $O-F$ distribution become less biased with a normal distribution around zero. 



(c) CTRL2 vs. GMI2

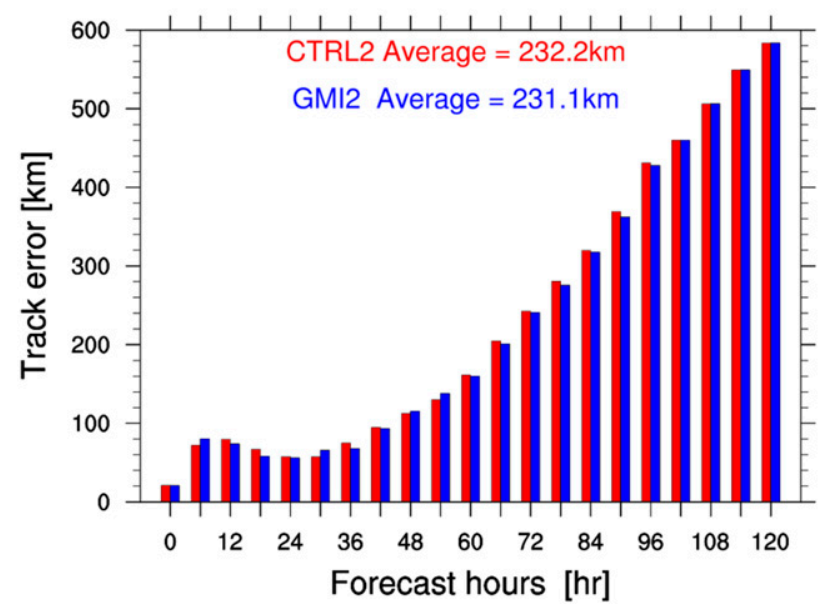

FIG. 9. Comparison of tracks between NHC best track (black curve) with 120-h HWRF forecasts (colored lines) initialized by each of the analyses generated in the 6 analysis-forecast cycles (as listed in the legend) for experiments without and with GM2 data assimilation for (a) CTRL2 and (b) GMI2. (c) The 120-h mean track error averaged over the all 6 forecasts in (a) and (b), for CTRL2 (red) and GMI2 (blue).

\section{Impact of GMI radiance data on numerical simulations of hurricanes}

\section{a. Track and intensity}

\section{1) Hurricane JoAquin}

The track forecast of Hurricane Joaquin in 2015 was challenging for the forecast community. As Joaquin reached category 3 strength and became a major hurricane on 1 October 2015, it made a hairpin turn and moved northeast under the influence of a deepening mid- to upper-level trough near the southeastern United States. As mentioned in the section 2c, the data assimilation experiments are performed from 0600 UTC 1 October to 1800 UTC 2 October (see Table 1) in 6-hourly analysis-forecast cycles. A 120 -h forecast is made at each analysis time of these 6-h analysis-forecast cycles after the data assimilation.

Figure 6 illustrates a comparison of the aggregated track forecasts between CTRL1 (without assimilation of GMI data) and GMI1 (with assimilation of GMI data) during the cycling periods from 0600 UTC 1 October to 1800 UTC 2 October 2015. From Fig. 6a, it is clear that the CTRL1 experiment shows systematic biases of forecast tracks toward the northwest side of the best track during the hairpin turn of Joaquin. These track errors are greatly reduced for experiments initiated after 0600 UTC 2 October, as the hairpin turn is close to completion. In contrast, GMI1 (Fig. 6b) also shows a great reduction in the magnitude of the track biases, owing to the assimilation of the GMI data. The largest difference in track forecasts between CTRL1 and GMI1 is found for the forecasts initiated at 0000 UTC 2 October. During the first 12-h forecasts, tracks from both the CTRL1 and GMI1 experiments show good agreement with the best track. However, the tracks in CTRL1 show a more northward trend soon after, while those in GMI1 attain a more realistic eastward propagation. Track errors of CTRL1 exceed $200 \mathrm{~km}$ after $30 \mathrm{~h}$ of forecast, while GMI1 retains a track error of less than $140 \mathrm{~km}$ throughout the $72-\mathrm{h}$ forecast period. 




Hours since 18 UTC 27 Sep 2016



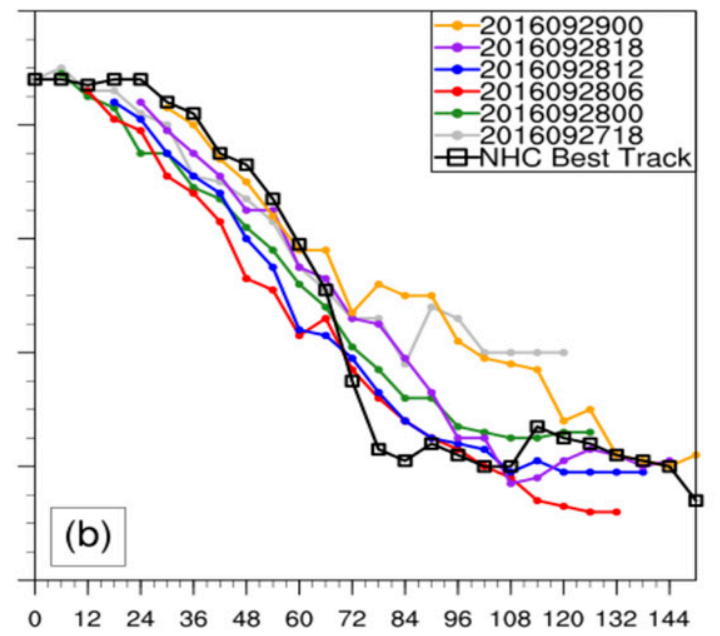

Hours since 18 UTC 27 Sep 2016

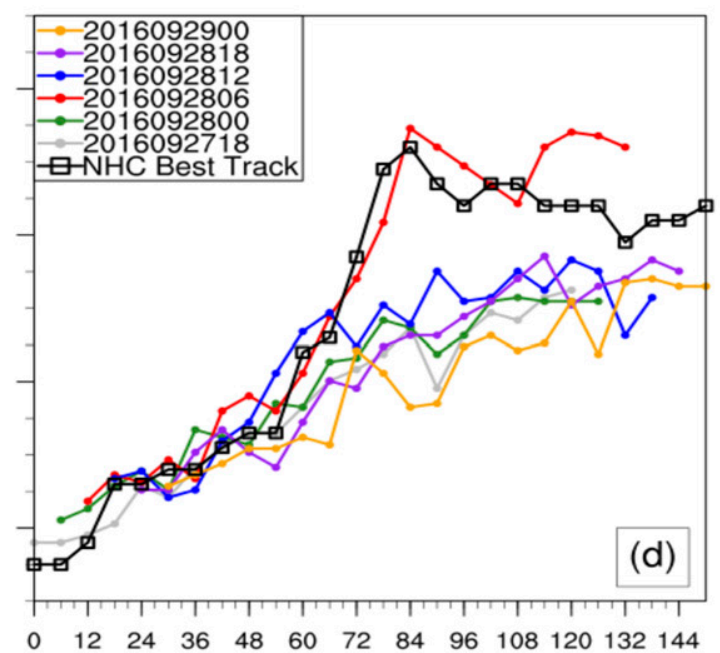

Hours since 18 UTC 27 Sep 2016
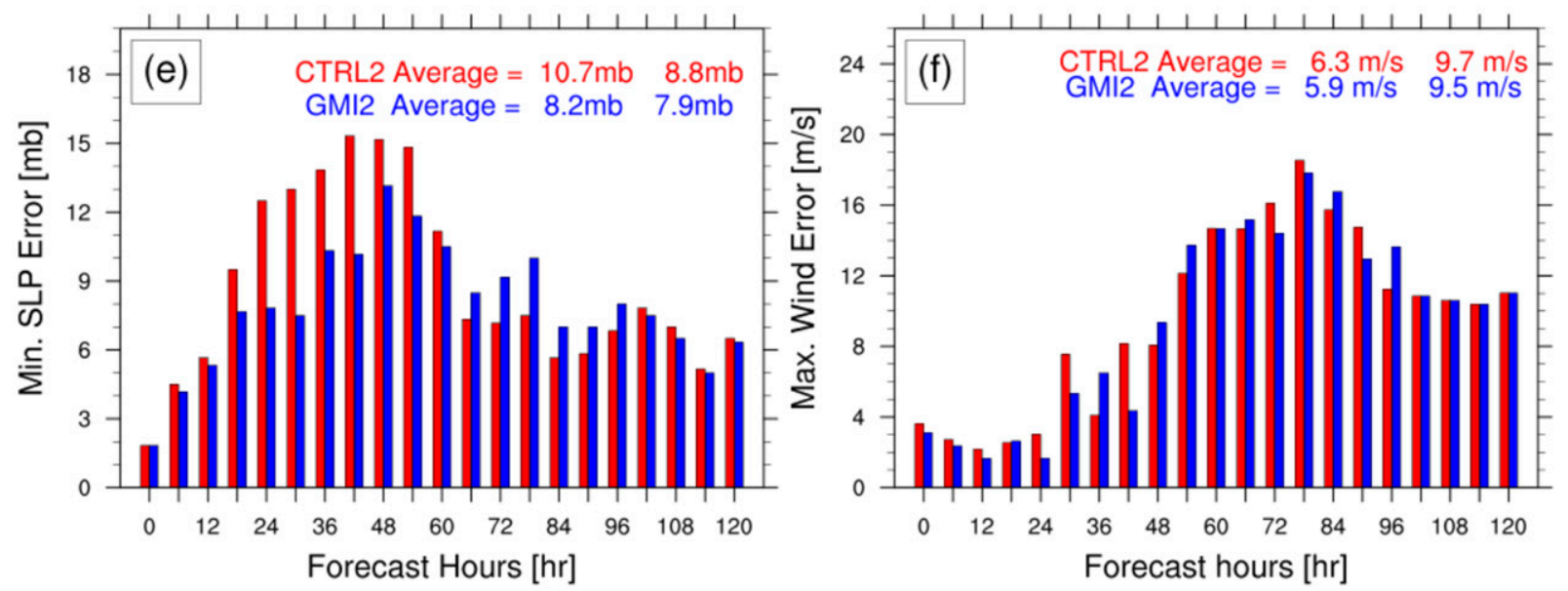

FIG. 10. Aggregated minimum SLP and maximum surface wind forecasts of (a),(c) CTRL2 and (b),(d) GMI2 (colored curves) compared with NHC best track data (black curve; 1800 UTC 27 Sep-0500 UTC Oct 2016) for the 120-h forecast started at the analysis time in all 6 analysis-forecast cycles (as listed in the legend). (c),(d) The mean forecast errors of minimum sea level pressure and maximum surface wind averaged over the forecasts started at the analysis time in all 6 analysis-forecast cycles (6-hourly from 1800 UTC 27 Sep to 0000 UTC 29 Sep 2016) for CTRL2 (red) and GMI2 (blue). In (e) and (f), the colored numbers on the left and right show the averaged errors over all 6 forecasts in first 60-h and 120-h HWRF integration, respectively. 


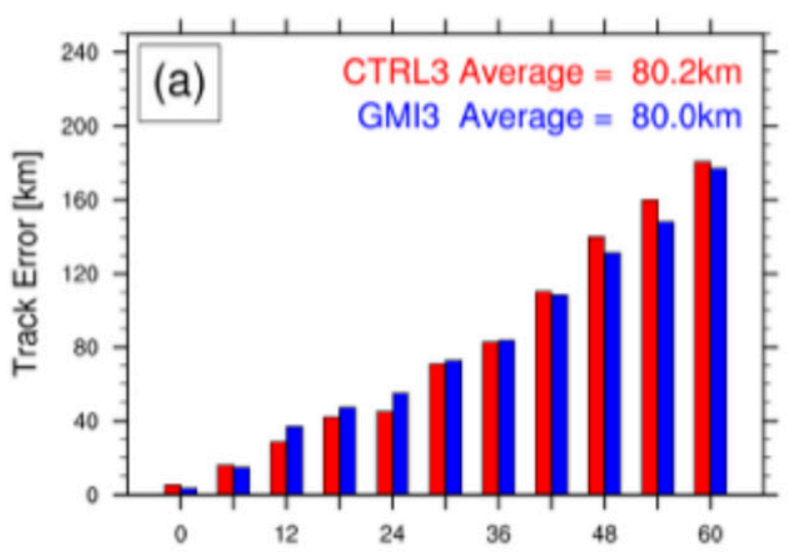

Forecast Lead Time [hr]
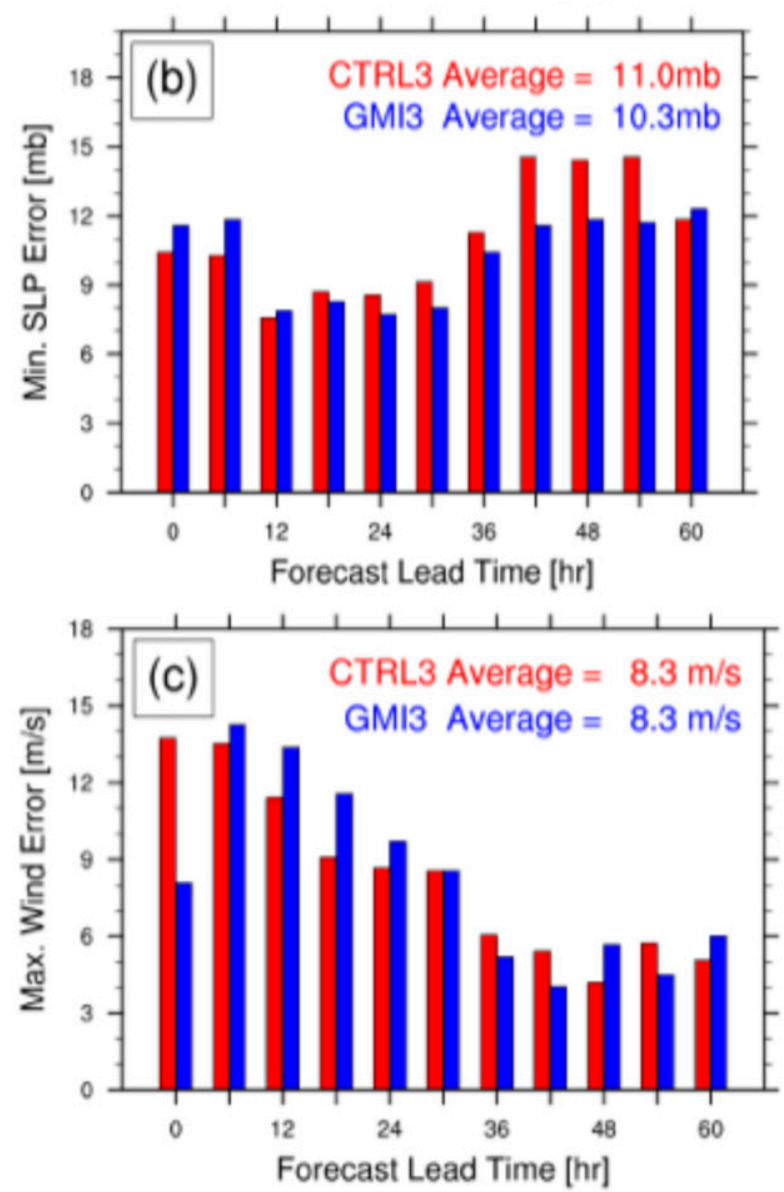

FIG. 11. 60-h mean forecast error of (a) track, (b) minimum sea level pressure, and (c) maximum surface wind averaged over forecasts started from the analysis time of all 7 analysis-forecast cycles for the mature phase of Matthew (6-hourly from 0000 UTC 3 October to 1200 UTC 4 October 2016) for CTRL3 (red) and GMI3 (blue).

The 120-h mean track error for CTRL1 and GMI1 (as shown in Figs. 6a and 6b), averaged over the forecasts started from each analysis of all 7 analysis-forecast cycles against the NHC best track data, is shown in Fig. 6c.
A consistent improvement in the track forecast is seen after the assimilation of GMI data, with roughly $20 \%$ and more than $12 \%$ reductions in track error during the first 72-h and over the entire 120-h forecasts, respectively.

Figure 7 shows a comparison of the minimum SLP and maximum surface wind forecasts between CTRL1 and GMI1 from all seven different forecast lead times. The figure indicated an overall positive or neutral impact of GMI data assimilation on the intensity forecast for Hurricane Joaquin over 120-h forecasts, while positive impacts on the intensity forecasts at first $60 \mathrm{~h}$ are evident.

Figures $8 \mathrm{a}$ and $8 \mathrm{~b}$ show the spatial distribution of assimilated brightness temperature from all microwave sensors (GMI data are highlighted in purple) over HWRF d01 at two different analysis times in GMI1. Except for GMI data, a set of microwave sounders, including the AMSU-A on board NOAA-15, NOAA-19, and Met $O p-A$ satellites, the MHS on board NOAA-18, $N O A A-19$, and $M e t O p-A$ satellites, and the ATMS is assimilated. In each of the times shown in Figs. 8a and $8 \mathrm{~b}$, GMI contains an overpass in the near-hurricane environment, where overpasses of other similar sensors are not present. This shows that the addition of GMI data to the entire pool of microwave sensors can provide more complete coverage of microwave satellite observations in the near-hurricane environment.

A comparison of GMI1 with CTRL1 shows that the impacts of assimilating GMI clear-sky radiance on the intensity forecast of Joaquin are less significant. The 60-h mean error in the maximum wind and minimum central sea level pressure (SLP) forecasts shows either neutral or marginal impacts (with less than $5 \%$ error reduction) from the assimilation of GMI data for most of the forecast time (figure not shown).

\section{2) Hurricane Matthew}

Compared to Joaquin, Hurricane Matthew exhibited less uncertainty in its track throughout its life cycle. Both the genesis and mature phases of Matthew are used to further examine the impact of assimilating GMI data on track and intensity forecasts (see the experimental design in Table 1). Figures 9 and 10 show comparisons of the track, minimum SLP, maximum wind forecasts between CTRL2 and GMI2 from different forecast lead times at the genesis, rapid intensification, and part of intensity change phases of Matthew from 1800 UTC 27 September to 0500 UTC October 2016. Figure 9 indicated a small, neutral impact of assimilation of GMI data on the track forecast of Hurricane Matthew. However, Fig. 10 reveals an obvious positive impact of GMI data assimilation on the intensity forecast with improvements in the first 60-h forecasts (up to 0000 UTC 

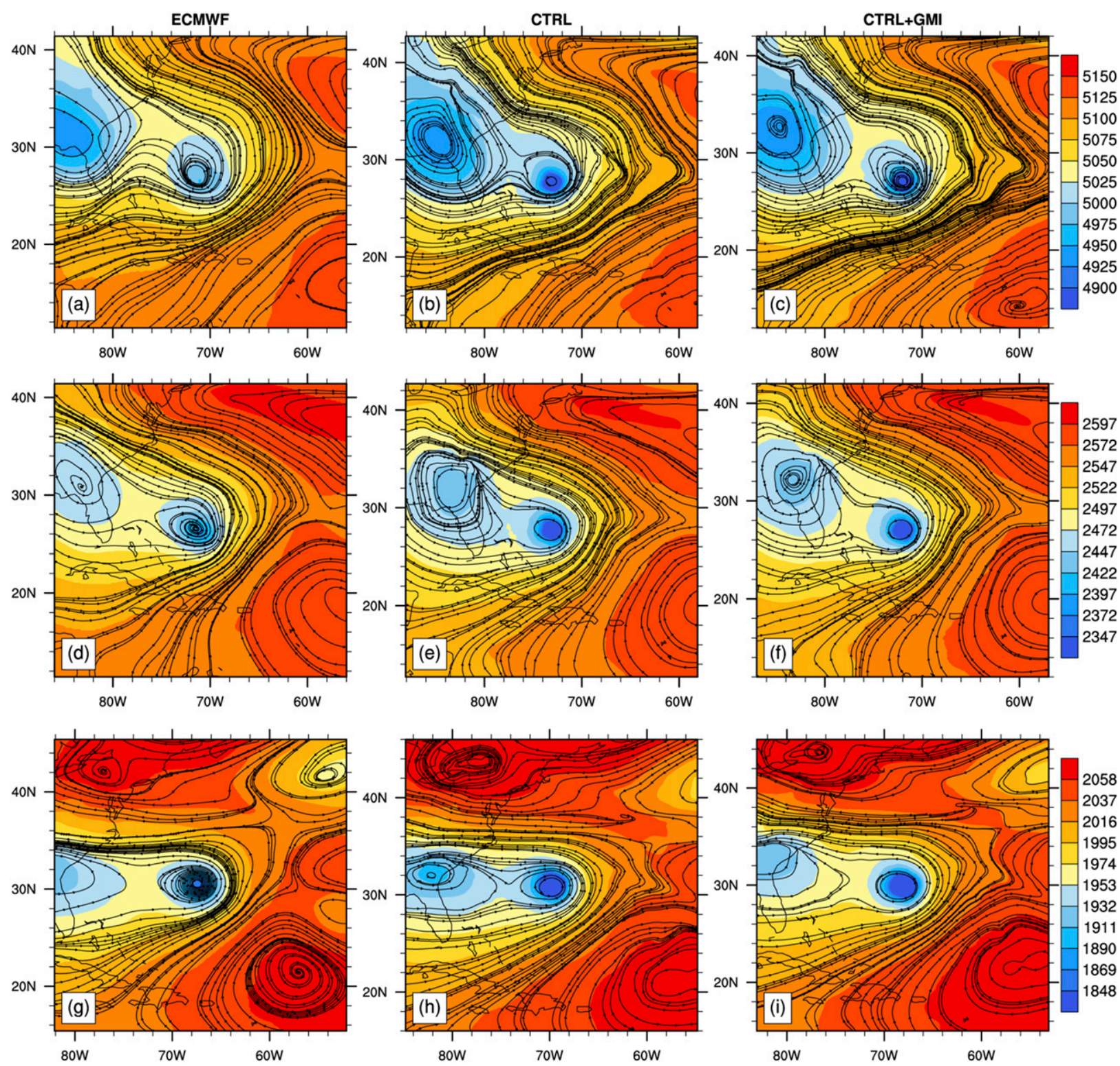

FIG. 12. Comparison of geopotential height (m) and streamline from (a),(d),(g) ERA-Interim reanalysis and (b),(c),(e),(f),(h),(i) HWRF forecasts. The HWRF 42-h forecasts valid at 1800 UTC 3 Oct 2016 at (b),(c) $550 \mathrm{mb}$ and (e),(f) $750 \mathrm{mb}$, compared with the corresponding ERA-Interim reanalysis. (h),(i) The 60-h HWRF forecasts valid at 1200 UTC 4 Oct 2016 at 800 mb, compared with the corresponding ERA-Interim reanalysis. The CTRL1 and GMI1 experiments (initiated at 0000 UTC 2 Oct) are shown in the middle and right panels, respectively.

1 October 2016). Specifically, it is apparent that CTRL2 (without GMI data assimilation) consistently undergoes rapid intensification too early, generally about $12-18 \mathrm{~h}$ ahead of the best track data (Fig. 10a). In contrast, GMI2 shows a correction in the forecast of minimum SLP (Fig. 10b) during the rapid intensification period. However, after 0000 UTC 1 October 2016, the end of Matthew's rapid intensification, the GMI data assimilation results in mixed forecast impacts with slightly degraded intensity forecast in some cases, reflecting the complicated dynamic and physical processes involved in the intensity changes. Nevertheless, the influences of the initial conditions on forecasts should not be as significant as the physical processes after $60-\mathrm{h}$ forecasts. Figure $10 \mathrm{c}$ displays the mean 120-h minimum SLP and maximum wind forecast errors. Overall, the assimilation of GMI data results in consistent positive impacts on both minimum SLP and maximum surface wind (MSW) for the first 60 -h forecast while some mixed impacts occur 
afterward, although the overall averaged impacts during the 120 -h forecast period are positive.

The mature phase of Matthew exhibits small uncertainty in both track and intensity forecasts. Figure 11 shows the 60-h mean track and intensity forecast, which covers the mature phase of Matthew but not the intensity change period afterward for experiments CTRL3 and GMI3. Overall, the assimilation of GMI clear-sky radiance has a neutral impact for the mature phase of Matthew.

\section{b. Impacts on hurricane large-scale environmental conditions}

Further diagnoses are conducted to demonstrate the impact of GMI data assimilation on the hurricane environment. Figure 12 shows geopotential height and streamline fields at $550 \mathrm{hPa}$ and $750 \mathrm{hPa}$ at the $42-\mathrm{h}$ forecasts for Joaquin from the CTRL1 and GMI1 experiments (valid at 1800 UTC 3 October 2015), respectively, compared with the corresponding ECMWFinterim reanalysis at the valid time. While the overall environmental features are similar between the CTRL1 and GMI1 experiments, noticeable differences are seen on the northwest side of the simulated vortices. Specifically, throughout the layer from 500 to $800 \mathrm{mb}$ $(1 \mathrm{mb}=1 \mathrm{hPa})$, the flow around the vortex in GMI 1 is more isolated from the deepening mid- to upper-level trough (on the northwest side of the hurricane) than that of CTRL, similar to a separation seen in the ECMWF reanalysis field.

\section{c. Impacts on forecasts of hurricane structure: Against dropsonde sounding during TCI}

To further verify the impact of GMI data assimilation on hurricane forecasts, independent observationsnamely, soundings obtained from a recent field program, the Tropical Cyclone Intensity (TCI) Experiment-are used. TCI is a collaborative field experiment sponsored by the Office of Naval Research (ONR), with the objective of improving forecasts of tropical cyclone intensity and structure changes (Doyle et al. 2017). During TCI, the NASA WB-57, which carries the High Definition Sounding System (HDSS) Dropsondes, is employed to sample the hurricane inner-core structure and its surrounding environment. The following verification utilizes dropsonde data collected during the mature phase of Joaquin from the HDSS.

Because many TCI soundings are taken close to the hurricane center (within $50 \mathrm{~km}$ of the center), choosing model profiles that have the same geophysical location as the sounding could result in significant overestimation of errors because of errors in the track forecast in each experiment. To account for this effect, model profiles in



FIG. 13. Locations and spatial distribution of model profiles and TCI sounding profiles (launched around 1800 UTC 2 Oct 2015, with distance less than $110 \mathrm{~km}$ from the hurricane center). Hurricane centers are shown by dotted circles.

each experiment are selected such that their positions relative to the center of the simulated vortices are the same as the position of the soundings relative to the best-track hurricane center. This guarantees that the focus of comparison is on the overall inner-core structure. Figure 13 shows the distribution of TCI soundings around 1800 UTC 2 October 2015 and the corresponding model verification grids (from 12-h forecasts initiated at 0600 UTC 2 October 2015). While the vortex center in GMI1 has a geophysical location similar to that of the best track data, CTRL1 has a track error of roughly $40 \mathrm{~km}$. Apparently, comparing soundings with model profiles at the same geophysical locations (indicated by black circles) is relatively unfair to CTRL1. Instead, model profiles that have the same relative positions with respect to the simulated vortices are more reasonable.

Figure 14 reveals the results of comparing the vertical profiles of root-mean-square error (RMSE) and bias in temperature and specific humidity against TCI dropsonde soundings. In the comparison, only soundings that are within $150 \mathrm{~km}$ of the hurricane center are included. The sample contains forecasts initiated at 0600 and 1200 UTC 2 October 2015, verified against TCI soundings launched around 1800 UTC 2 October and 1800 UTC 3 October, giving a total sample size of 156. From the RMSE in Figs. 14a and 14b, the assimilation of GMI clear-sky radiance reduces error in temperature throughout the entire troposphere, while its impact on specific humidity lies mostly in the midtroposphere 

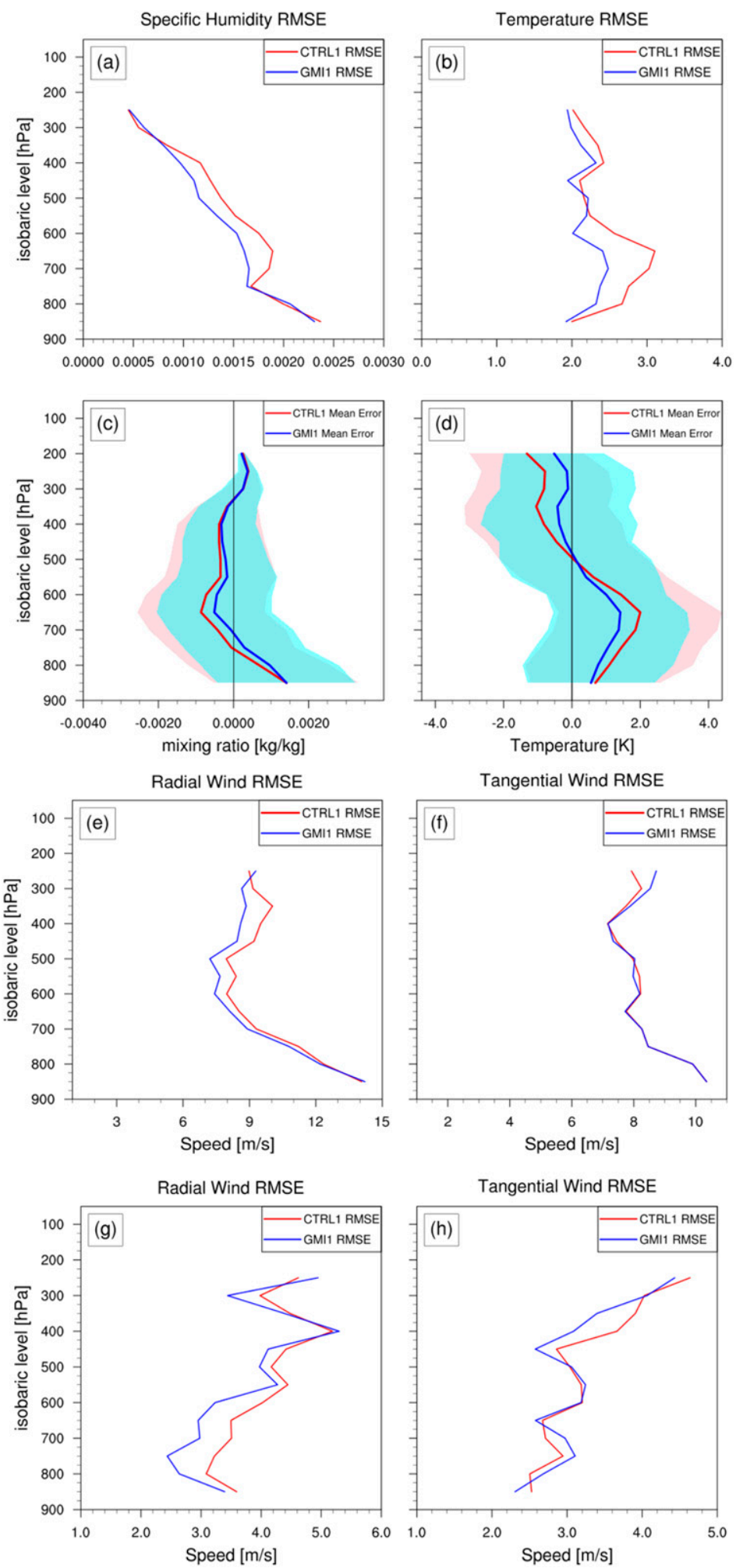

FIG. 14. Vertical profiles of (a),(b) RMSE and (c),(d) mean bias (solid colored lines) and standard deviation (shading) of specific humidity and temperature forecasts and RMSEs of (e) radial and (f) tangential wind forecasts (initiated at 0600 and 1200 UTC 2 Oct 2015) for CTRL1 (red) and GMI1 (blue). Each of the 


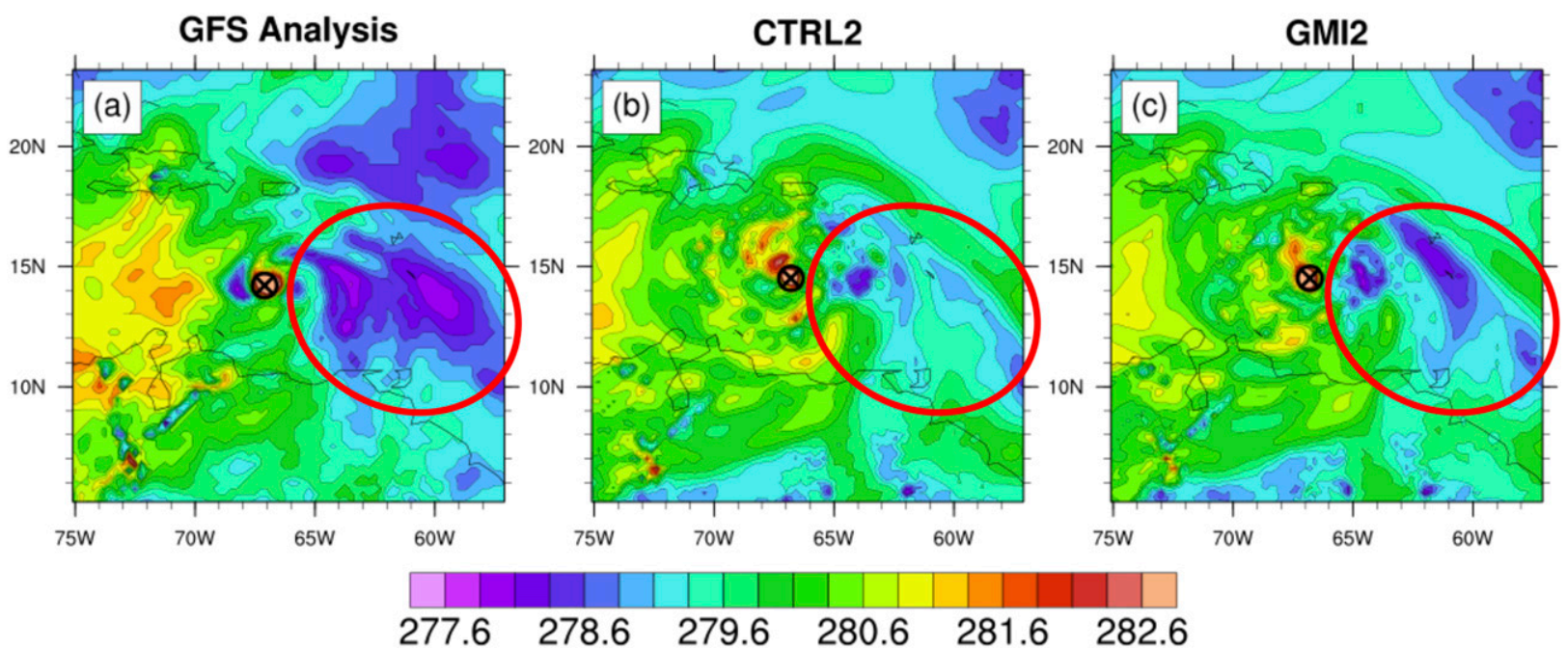

FIG. 15. Comparison of 30-h temperature (K) forecasts at $650 \mathrm{mb}$, valid at 1800 UTC 29 Sep 2016 for (b) CTRL2 and (c) GMI2 against (a) GFS analysis at the corresponding time. Hurricane centers are indicated by the crossed circles. Red circles indicate the locations of the large pool of cold air to the east of storm.

(400-700 mb; Fig. 14b). Figures $14 \mathrm{c}$ and $14 \mathrm{~d}$ show the mean bias and the standard deviation of temperature and specific humidity at different pressure levels. For temperature, both experiments (CTRL1 and GMI1) produce warm biases in the lower troposphere (below $500 \mathrm{hPa}$ ) and cold bias in the upper troposphere (Fig. 14c). However, these biases in GMI1 are noticeably smaller than that in CTRL1. For specific humidity, GMI data also result in reduction of the biases.

The impact of GMI on the wind field is also examined. Figures $14 \mathrm{e}-\mathrm{h}$ show the comparison of RMSE for radial wind and tangential wind between CTRL1 and GMI1 verified against TCI soundings, respectively. Within a $150-\mathrm{km}$ radius of the hurricane center, the wind magnitudes from both CTRL1 and GMI1 are greater than the dropsonde data at the lower levels (below 700-hPa pressure levels), while GMI data assimilation improves the radial wind fields and results in a neutral impact on tangential winds in the middle and upper levels (Figs. 14e,f). The comparison is also revealed on the near-hurricane environment within a radius of between 150 and $600 \mathrm{~km}$ from the center, giving a sample size of 114. From Figs. $14 \mathrm{~g}$ and $14 \mathrm{~h}$, it is found that assimilation of the GMI data results in noticeable improvements in the radial wind fields in both the lower troposphere (between 600 and
$800 \mathrm{mb}$ ) and upper troposphere (about $300 \mathrm{mb}$ ), as shown in Fig. 14g. In terms of tangential velocity (Fig. 14h), the impact of GMI is rather neutral, with some positive impact on the midlevel troposphere (between 350 and $450 \mathrm{mb}$ ).

\section{d. Impact on temperature and moisture fields during the genesis of Matthew}

Assimilation of GMI clear-sky radiance is found to provide noticeable improvements in the intensity forecast of the genesis phase of Matthew. During this phase, it is found that widespread cold air dominates the middle to lower troposphere in the east sector of the storm, as shown by the GFS analysis between 650 and $850 \mathrm{mb}$. Figure 15 compares HWRF 30 -h forecasts valid at 0800 UTC 29 September 2016 against the corresponding GFS analysis. It shows that both CTRL2 and GMI2 have a similar east-west temperature gradient across the storm in the midtroposphere. However, in CTRL2 the cold temperature on the east side of the storm is noticeably weaker compared to the GFS analysis. In contrast, GMI2 has more dominant cold air accumulated in the east sector of the storm, resembling the cold air configuration seen in the GFS analysis, even though the magnitude and spread are still underestimated.

forecasts is verified against TCI soundings launched within a $150-\mathrm{km}$ radius of the hurricane center around 1800 UTC 2 Oct and 1800 UTC 3 Oct 2015, giving a sample size of 156 . As in (e) and (f), the RMSE of (g) radial and (h) tangential wind forecasts are verified against TCI soundings launched between 150 and $600 \mathrm{~km}$ from hurricane centers, giving a sample size of 114 . 

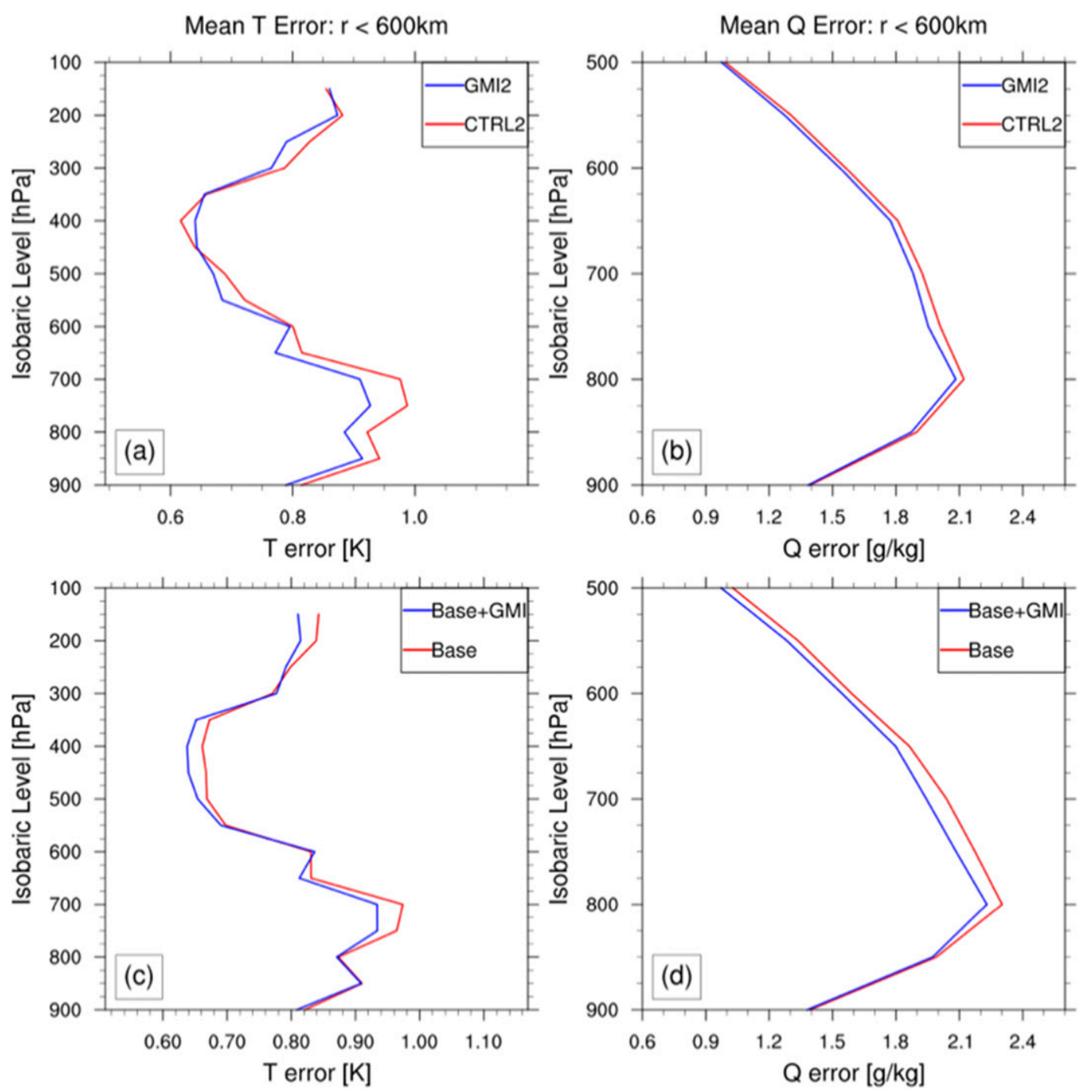

FIG. 16. RMSE for 30-h forecasts of (a) temperature and (b) specific humidity, verified against GFS analysis. Statistics are averaged over forecasts started from the analysis time of all 6 analysis-forecast cycles (6-hourly from 1800 UTC 27 Sep to 0000 UTC 29 Sep 2016 during the genesis phase of Matthew) within a 600-km radius of the hurricane centers (blue: CTRL2 and Base; red: GMI2 and Base+GMI).

Figure 16 shows the vertical profiles of RMSE for temperature and specific humidity within a $600-\mathrm{km}$ radius of the observed storm center, averaged over 30 -h forecasts from each analysis of all analysis-forecast cycles of the genesis of Matthew (CTRL2 and GMI2). For the errors in the temperature field, assimilation of GMI data has a positive impact throughout almost the entire troposphere, especially below the $600-\mathrm{hPa}$ pressure level. For specific humidity, obvious error reduction can be seen around the 500-hPa pressure level.

In fact, during the genesis phase of Matthew, GMI overpasses are overlapped with other microwave sensors that have similar humidity profiling channels, including AMSU-A, MHS, and ATMS, as shown in Figs. 8c and 8d.
These sensors have humidity sensor channels similar to those of GMI near 23.8 and $183 \mathrm{GHz}$. To further investigate the impact of GMI on HWRF forecasts in this type of case, another set of experiments is conducted, called Base and Base +GMI in Table 1. The Base is similar to CTRL2, expect no satellite observations are assimilated. GMI clear-sky radiance is assimilated in Base + GMI, along with other data are assimilated into the Base, including conventional observations, GPS-RO data, and satellite-retrieved wind. In addition, considering the early results of CTRL2 and GMI2 (Fig. 10), the evaluation of forecast impacts is conducted for the first 60-h forecasts to emphasize Matthew's genesis and rapid intensification phases only, and also to obtain the clear 

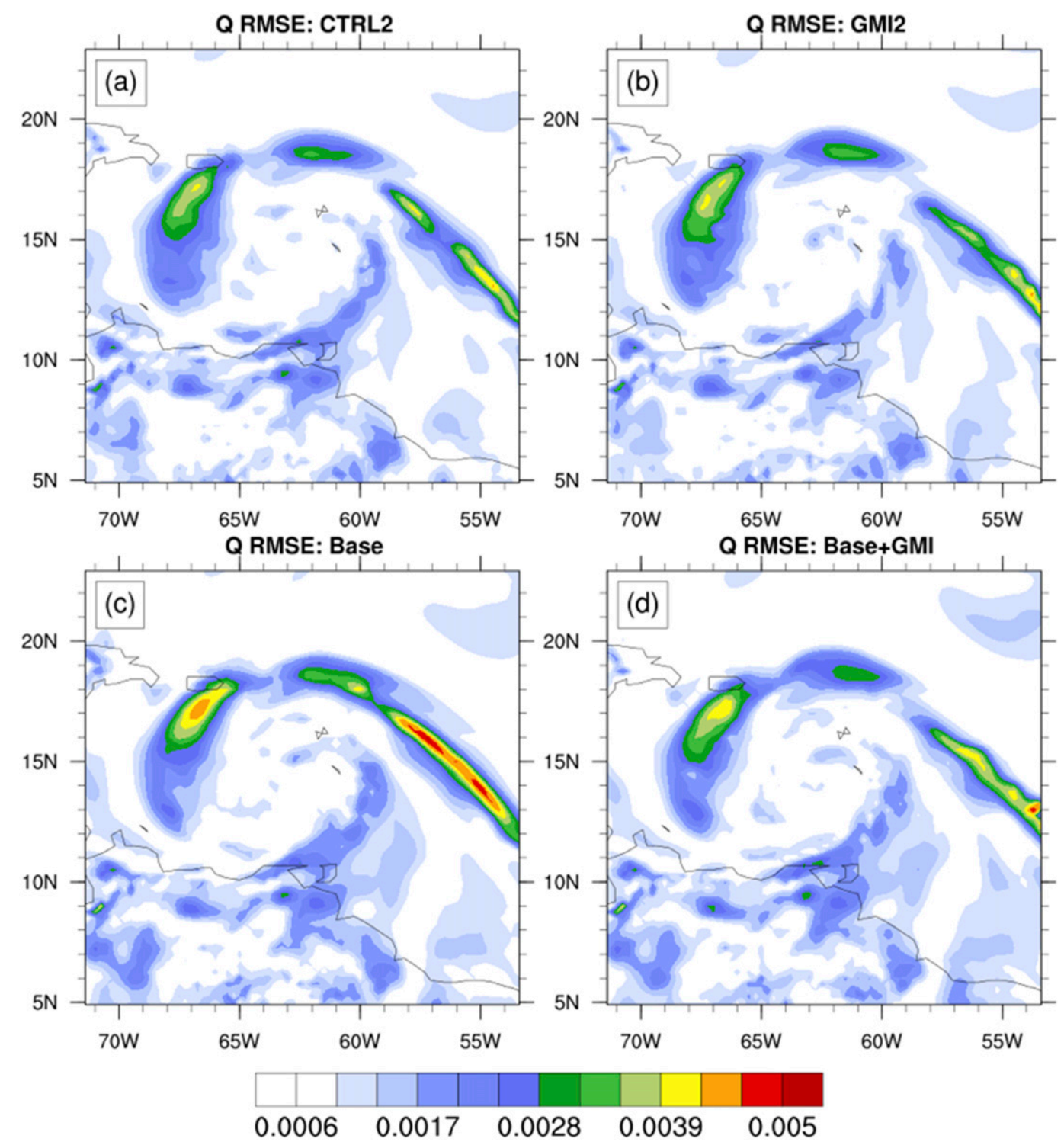

FIG. 17. Averaged 500-850-mb RMSE of specific humidity at 30-h forecast (valid at 0000 UTC 29 Oct 2016). (a) CTRL2, (b) GMI2, (c) Base, and (d) Base+GMI. RMSE is calculated based on forecasts verified against GFS analyses at the valid time.

influences of data assimilation (e.g., initial conditions) that are commonly best shown in the short-range forecasts.

Figure 17 shows a comparison of 500-850-mb averaged RMSE of specific humidity forecasts between CTRL2, GMI2, Base, and Base+GMI. Figures 17a and 17b show that CTRL2 and GMI2 have a rather similar spatial distribution of specific humidity RMSE. Clearly, assimilating GMI clear-sky radiance provides only a small improvement in the moisture field, which is consistent with the result shown in Fig. 17b. In Fig. 17c, when no radiance observation is assimilated, the Base experiment shows the largest error in the moisture field among all 4 experiments, especially on both the northeast and northwest sides of the storm. When GMI data are added, Base+GMI shows a noticeable reduction in RMSE in specific humidity compared to the Base experiment, as shown in Fig. 17d, which reveals the sole impact of GMI data assimilation on the specific humidity when other similar satellite sensors are not present. In addition, a comparison of Fig. $17 \mathrm{~d}$ with Figs. $17 \mathrm{a}$ and $17 \mathrm{~b}$ shows that the overall spatial distribution of RMSE in Base +GMI is similar to that in CTRL2 and GMI2, indicating that the constraints imposed by GMI data assimilation on specific humidity are consistent with other sensors, hence resulting in a similar improvement. 



FIG. 18. The 60-h mean error of (a) track, (b) minimum sea level pressure, and (c) maximum surface wind, averaged over forecasts started from each analysis time of all 6 analysis-forecast cycles of the genesis phase of Matthew (6-hourly from 1800 UTC 27 Sep to 0000 UTC 29 Sep 2016), for Base (red) and Base+GMI (blue).

Figure 18 shows the mean errors for minimum SLP, MSW, and track forecasts in Base and Base + GMI, averaged over the forecasts from the analyses of all the analysisforecast cycles. It is found that without the presence of other sensors, the assimilation of GMI clear-sky radiance alone is also able to provide similar positive impacts on HWRF forecasts during Matthew's genesis phase.

\section{Summary and conclusions}

This study examines the impact of assimilating GMI clear-sky radiance on HWRF hurricane forecasts using the GSI-based hybrid ensemble-3DVar data assimilation system. Prior to assimilating the radiance, bias corrections are conducted using a two-step approach. First, a static bias correction is used to derive a rough estimate of the bias correction coefficient using regression on a representative sample of radiance departure. The estimated bias correction coefficients are then treated as a first guess to go through a series of iterative procedures using the variational bias correction capability in GSI to capture the small-time variation of the coefficients.

Hurricane Joaquin in 2015 and Matthew in 2016 are used as case studies to evaluate the impact of assimilating GPM GMI clear-sky radiance on hurricane analysis and forecasts. For the mature phase of Joaquin, assimilating GMI radiance results in significant improvement in the track forecast, especially during its hairpin turn. Comparing against ERA-Interim data, we found a significant improvement in the forecast of the geopotential height field in the near-hurricane environment, leading to a more realistic interaction between the simulated hurricane vortex and the nearby mid- to upper-level trough. The overall impact on the intensity forecast of the mature phase of Joaquin is significant in short range $(60 \mathrm{~h})$ but relatively modest over 120 -h forecasts. This is mostly due to the fact that clear-sky radiance observations occur away from the inner-core region of the hurricane.

For the genesis phase of Matthew, forecast results show that assimilating GMI radiance improves the intensity forecast, especially during the first 48-60-h forecast. Close examination of the forecast result using GFS analysis shows that assimilating GMI clear-sky radiance improves the forecast of mid- to lower-level cold air aggregated on the northeast side of the storm, which causes Matthew's intensification to slow down. Using GFS analysis as reference, the overall RMSE statistics show a clear improvement in temperature throughout the entire troposphere and in low- to midlevel specific humidity in the near-hurricane environment (radius less than $500 \mathrm{~km}$ ), even when other microwave sounders are present (e.g., AMSU-A, ATMS, MHS, etc.).

An additional experiment is performed for the mature phases of Matthew, during which Matthew exhibits relatively small uncertainty in both track and intensity. The overall result of this phase shows that while the impact 
of GMI on both track and intensity is rather small, no negative impact is seen.

The experiments performed in this study indicate that there is a great potential to assimilate GMI satellite radiances into the regional hurricane prediction models (e.g., HWRF) to improve the operational hurricane prediction. However, numerical experiments from this paper did not fully follow the operational HWRF analysis and forecast procedures (e.g., no tail Doppler radar data assimilation, no vortex intensity correction, with the emphasis on shortrange forecasts only, etc.). Meanwhile, the operational HWRF Model has been updated at the time when this paper was written. Therefore, future work will further examine the impacts of GMI data assimilation on operational HWRF hurricane analyses and forecasts and achieve the statistical significance. The investigation will also extend to assimilating GMI all-sky radiance, especially in the hurricane inner-core regions. Moreover, although the two-step bias correction methods in this study shed light on bias correction in the regional model, additional efforts are still required to address the issue adequately in the hurricane environment.

Acknowledgments. This study is supported by the NOAA Joint Center for Satellite Data Assimilation (JCSDA) through research Grant NA15NES4400004 and NASA Award NNX17AE95G. The first author $(\mathrm{Pu})$ is also supported by the Office of Naval Research Award N000141612491. Computer resources provided by the NOAA S4 computer system and Jet supercomputer system, and the University of Utah's Center for High-Performance Computing (CHPC) are greatly appreciated. Review comments from four reviewers and Dr. Altug Aksoy (editor) were useful for improving the overall manuscript.

\section{REFERENCES}

Aravéquia, J. A., I. Szunyogh, E. J. Fertig, E. Kalnay, D. Kuhl, and E. J. Kostelich, 2011: Evaluation of a strategy for the assimilation of satellite radiance observations with the local ensemble transform Kalman filter. Mon. Wea. Rev., 139, 1932-1951, https://doi.org/10.1175/2010MWR3515.1.

Bauer, P., P. Lopez, A. Benedetti, D. Salmond, and E. Moreau, 2006: Implementation of 1D4D-Var assimilation of precipitation affected microwave radiances at ECMWF. I: 1D-Var. Quart. J. Roy. Meteor. Soc., 132, 2277-2306, https://doi.org/10.1256/qj.05.189.

Biswas, M. K., L. Carson, C. Holt, and L. Bernardet, 2015: Community HWRF Users' Guide V3.7a. Development Testbed Center, 152 pp., http://www.dtcenter.org/HurrWRF/users/docs/ users_guide/HWRF_v3.7a_UG.pdf.

Dee, D. P., 2004: Variational bias correction of radiance data in the ECMWF system. Proc. ECMWF Workshop on Assimilation of High Spectral Resolution Sounders in NWP, Reading, United Kingdom, ECMWF, 97-112.

Derber, J. C., and W.-S. Wu, 1998: The use of TOVS cloud-cleared radiances in the NCEP SSI analysis system. Mon. Wea. Rev.,
126, 2287-2299, https://doi.org/10.1175/1520-0493(1998) $126<2287$ : TUOTCC $>2.0$. CO; 2 .

Doyle, J., and Coauthors, 2017: A view of tropical cyclones from above: The Tropical Cyclone Intensity (TCI) experiment. Bull. Amer. Meteor. Soc., 98, 2113-2134, https://doi.org/10.1175/ BAMS-D-16-0055.1.

Garrett, K., L. Moy, F. Iturbide-Sanchez, and S.-A. Boukabara, 2010: A physically-based rainfall rate algorithm for the Global Precipitation Mission. Fifth IPWG Workshop, Hamburg, Germany, IPGW. 15 pp., http://www.isac.cnr.it/ ipwg/meetings/ hamburg-2010/pres/Garrett.pdf.

Goerss, J., 2009: Impact of satellite observations on the tropical cyclone track forecasts of the NOGAPS. Mon. Wea. Rev., 137, 41-50, https://doi.org/10.1175/2008MWR2601.1.

Gopalakrishnan, S. G., F. Marks, X. Zhang, J.-W. Bao, K.-S. Yeh, and R. Atlas, 2011: The Experimental HWRF System: A study on the influence of horizontal resolution on the structure and intensity changes in tropical cyclones using an idealized framework. Mon. Wea. Rev., 139, 1762-1784, https://doi.org/ 10.1175/2010MWR3535.1.

Han, Y., P. van Delst, Q. Liu, F. Weng, B. Yan, R. Treadon, and J. Derber, 2006: JCSDA Community Radiative Transfer Model (CRTM) - version 1. NOAA Tech. Rep., NESDIS 122, $40 \mathrm{pp}$.

Hoppel, K. W., S. D. Eckermann, L. Coy, G. E. Nedoluha, D. R. Allen, S. D. Swadley, and N. L. Baker, 2013: Evaluation of SSMIS upper atmosphere sounding channels for high altitude data assimilation. Mon. Wea. Rev., 141, 3314-3330, https:// doi.org/10.1175/MWR-D-13-00003.1.

Hou, A. Y., and Coauthors, 2014: The Global Precipitation Measurement mission. Bull. Amer. Meteor. Soc., 95, 701-722, https://doi.org/10.1175/BAMS-D-13-00164.1.

Kazumori, M., 2014: Satellite radiance assimilation in the JMA operational mesoscale 4DVAR system. Mon. Wea. Rev., 142, 1361-1381, https://doi.org/10.1175/MWR-D-13-00135.1.

Lin, H., S. S. Weygandt, S. G. Benjamin, and M. Hu, 2017: Satellite radiance data assimilation within the hourly updated Rapid Refresh. Wea. Forecasting, 32, 1273-1287, https://doi.org/ 10.1175/WAF-D-16-0215.1.

Liu, Q., N. Surgi, S. Lord, W.-S. Wu, S. Parrish, S. Gopalakrishnan, J. Waldrop, and J. Gamache, 2006: Hurricane initialization in HWRF model. 27th Conf. on Hurricanes and Tropical Meteorology, Monterey, CA, Amer. Meteor. Soc., 8A.2, https://ams. confex.com/ams/27Hurricanes/techprogram/paper_108496.htm.

Liu, Z., C. S. Schwartz, C. Snyder, and S.-Y. Ha, 2012: Impact of assimilating AMSU-A radiances on forecasts of 2008 Atlantic tropical cyclones initialized with a limited-area ensemble Kalman filter. Mon. Wea. Rev., 140, 4017-4034, https://doi.org/ 10.1175/MWR-D-12-00083.1.

McNally, P., J. C. Derber, W. Wu, and B. B. Katz, 2000: The use of TOVS level-1b radiances in the NCEP SSI analysis system. Quart. J. Roy. Meteor. Soc., 126, 689-724, https://doi.org/ 10.1002/qj.49712656315.

Migliorini, S., 2012: On the equivalence between radiance and retrieval assimilation. Mon. Wea. Rev., 140, 258-265, https:// doi.org/10.1175/MWR-D-10-05047.1.

Miyoshi, T., and Y. Sato, 2007: Assimilating satellite radiances with a local ensemble transform Kalman filter (LETKF) applied to the JMA global model (GSM). SOLA, 3, 37-40, https://doi.org/ 10.2151/sola.2007-010.

Okamoto, K., and J. C. Derber, 2006: Assimilation of SSM/I radiances in the NCEP global data assimilation system. Mon. Wea. Rev., 134, 2612-2631, https://doi.org/10.1175/MWR3205.1. 
Pu, Z., and L. Zhang, 2010: Validation of AIRS temperature and moisture profiles over tropical oceans and their impact on numerical simulations of tropical cyclones. J. Geophys. Res., 115, D24114, https://doi.org/10.1029/2010JD014258.

, W.-K. Tao, S. Braun, J. Simpson, Y. Jia, J. Halverson, W. Olson, and A. Hou, 2002: The impact of TRMM data on mesoscale numerical simulation of Supertyphoon Paka. Mon. Wea. Rev., 130, 2448-2458, https://doi.org/10.1175/1520-0493 (2002) $130<2448$ :TIOTDO > 2.0.CO;2.

$\longrightarrow$ X. Li, C. Velden, S. Aberson, and W. T. Liu, 2008: Impact of aircraft dropsonde and satellite wind data on the numerical simulation of two landfalling tropical storms during TCSP. Wea. Forecasting, 23, 62-79, https://doi.org/10.1175/2007WAF2007006.1.

- S. Zhang, M. Tong, and V. Tallapragada, 2016: Influence of the self-consistent regional ensemble background error covariance on hurricane inner-core data assimilation with the GSI-based hybrid system for HWRF. J. Atmos. Sci., 73, 49114925, https://doi.org/10.1175/JAS-D-16-0017.1.

Qin, Z., X. Zou, and F. Weng, 2013: Evaluating added benefits of assimilating GOES imager radiance data in GSI for coastal QPFs. Mon. Wea. Rev., 141, 75-92, https://doi.org/10.1175/ MWR-D-12-00079.1.

Sakamoto, M., and J. R. Christy, 2009: The influences of TOVS radiance assimilation on temperature and moisture tendencies in JRA-25 and ERA-40. J. Atmos. Oceanic Technol., 26, 1435-1455, https://doi.org/10.1175/2009JTECHA1193.1.

Tallapragada, V., and Coauthors, 2015: Hurricane Weather Research and Forecasting (HWRF) Model: 2015 scientific documentation. NCAR Development Testbed Center Rep., 119 pp., http://nldr.library.ucar.edu/collections/technotes/ TECH-NOTE-000-000-000-893.pdf.

Wang, P., and Coauthors, 2015: Assimilation of thermodynamic information from advanced infrared sounders under partially cloudy skies for regional NWP. J. Geophys. Res. Atmos., 120, 5469-5484, https://doi.org/10.1002/2014JD022976.

Weng, F., 2007: Advances in radiative transfer modeling in support of satellite data assimilation. J. Atmos. Sci., 64, 3799-3807, https://doi.org/10.1175/2007JAS2112.1.

Wu, T.-C., M. Zupanski, L. D. Grasso, P. J. Brown, C. D. Kummerow, and J. A. Kna, 2016: The GSI capability to assimilate TRMM and GPM hydrometeor retrievals in HWRF. Quart. J. Roy. Meteor. Soc., 142, 2768-2787, https:// doi.org/10.1002/qj.2867.

Xu, D., Z. Liu, X.-Y. Huang, J. Min, and H. Wang, 2013: Impact of assimilating IASI radiance observations on forecasts of two tropical cyclones. Meteor. Atmos. Phys., 122, 1-18, https://doi.org/ 10.1007/s00703-013-0276-2.

_ J. Min, F. Shen, J. Ban, and P. Chen, 2016: Assimilation of MWHS radiance data from the FY-3B satellite with the WRF Hybrid-3DVAR system for the forecasting of binary typhoons. J. Adv. Model. Earth Syst., 8, 1014-1028, https://doi.org/10.1002/ 2016MS000674.

Yang, C., Z. Liu, J. Bresch, S. R. H. Rizvi, X.-Y. Huang, and J. Min, 2016: AMSR2 all-sky radiance assimilation and its impact on the analysis and forecast of Hurricane Sandy with a limited-area data assimilation system. Tellus, 68A, 30917, https://oi.org/ 10.3402/tellusa.v68.30917.

Zhang, H., and Z. Pu, 2014: Influence of assimilating surface observations on the prediction of landfalls of Hurricane Katrina (2005) with ensemble Kalman filter. Mon. Wea. Rev., 142, 2915-2934, https://doi.org/10.1175/MWR-D-14-00014.1.

Zhang, M., M. Zupanski, M.-J. Kim, and J. A. Knaff, 2013: Assimilating AMSU-A radiances in the TC core area with NOAA operational HWRF (2011) and a hybrid data assimilation system: Danielle (2010). Mon. Wea. Rev., 141, 3889-3907, https:// doi.org/10.1175/MWR-D-12-00340.1.

Zhang, S. Q., M. Zupanski, A. Y. Hou, X. Lin, and S. H. Cheung, 2013: Assimilation of precipitation-affected radiances in a cloud-resolving WRF ensemble data assimilation system. Mon. Wea. Rev., 141, 754-772, https://doi.org/10.1175/ MWR-D-12-00055.1.

Zhang, S., Z. Pu, and C. Velden, 2018: Impact of enhanced atmospheric motion vectors on HWRF hurricane analyses and forecasts with different data assimilation configurations. Mon. Wea. Rev., 146, 1549-1569, https://doi.org/10.1175/MWR-D17-0136.1.

Zhu, Y., J. Derber, A. Collard, D. Dee, R. Treadon, G. Gayno, and J. A. Jung, 2014: Enhanced radiance bias correction in the National Centers for Environmental Prediction's Gridpoint Statistical Interpolation data assimilation system. Quart. J. Roy. Meteor. Soc., 140, 1479-1492, https://doi.org/10.1002/qj.2233.

Zou, X., Z. Qin, and F. Weng, 2011: Improved coastal precipitation forecasts with direct assimilation of GOES-11/12 imager radiances. Mon. Wea. Rev., 139, 3711-3729, https://doi.org/ 10.1175/MWR-D-10-05040.1.

—, F. Weng, B. Zhang, L. Lin, Z. Qin, and V. Tallapragada, 2013: Impacts of assimilation of ATMS data in HWRF on track and intensity forecasts of 2012 four landfall hurricanes. J. Geophys. Res. Atmos., 118, 11 558-11 576, https://doi.org/10.1002/ 2013JD020405. 